\title{
Disclosing the spatiotemporal structure of parametric down-conversion entanglement through frequency up-conversion
}

\author{
E. Brambilla, ${ }^{1, *}$ O. Jedrkiewicz, ${ }^{1,2}$ L. A. Lugiato, ${ }^{1}$ and A. Gatti ${ }^{1,2}$ \\ ${ }^{1}$ CNISM and Dipartimento di Scienze Fisiche e Matematiche, Università dell' Insubria, Via Valleggio 11, I-22100 Como, Italy \\ ${ }^{2}$ CNR, Istituto di Fotonica e Nanotecnologie, Piazza Leonardo da Vinci 4, Milano, Italy
}

(Received 27 April 2012; published 25 June 2012)

\begin{abstract}
In this work we propose and analyze a scheme where the full spatiotemporal correlation of twin photons or beams generated by parametric down-conversion is detected by using its inverse process, i.e., sum frequency generation. Our main result is that, by imposing independently a temporal delay $\Delta t$ and a transverse spatial shift $\Delta x$ between two twin components of PDC light, the up-converted light intensity provides information on the correlation of the PDC light in the full spatiotemporal domain and should enable the reconstruction of the peculiar X-shaped structure of the correlation predicted previously [A. Gatti et al. Phys. Rev. Lett. 102, 223601 (2009); L. Caspani et al. Phys. Rev. A 81, 033808 (2010); E. Brambilla et al. Phys. Rev. A 82, 013835 (2010)]. Through both a semianalytical and a numerical modeling of the proposed optical system, we analyze the feasibility of the experiment and identify the best conditions to implement it. In particular, the tolerance of the phase-sensitive measurement against the presence of dispersive elements, imperfect imaging conditions, and possible misalignments of the two crystals is evaluated.
\end{abstract}

DOI: 10.1103/PhysRevA.85.063834

\section{INTRODUCTION}

Recent theoretical investigations [1-3] outlined a peculiar spatiotemporal geometry of the biphoton correlation characterizing the entanglement of twin beams generated by parametric down-conversion (PDC) from a broad quasimonochromatic pump pulse. In collinear phase-matching conditions, the biphotonic correlation displays an $\mathrm{X}$-shaped geometry as a function of the relative spatial and temporal coordinates. This structure is nonfactorable in space and time, thus offering the relevant possibility of manipulating the temporal bandwidth of the entanglement of twin photons by acting on their spatial degrees of freedom. The term "X entanglement" was used [1] to describe this geometry. A key feature that emerged was the extreme spatial and temporal localization of the biphotonic correlation, in the micrometer and femtosecond range, respectively, which is present only when twin photons are detected in the near field of the PDC source. This feature allows, in principle, the generation of ultrabroadband temporally entangled photons, via the proper control of their spatial degrees of freedom. We also showed that these features of $\mathrm{X}$ entanglement persist in the high-gain regime of PDC, where stimulated down-conversion becomes the main source of twin photon pairs. A detailed study can be found in Refs. [1] and [2] for type I phase matching and in Ref. [3] for type II phase matching.

$\mathrm{X}$ entanglement expresses a strong coupling between the temporal delay and the transverse spatial separation of the twin photons at the crystal output face. This coupling in direct space is connected to the nonfactorability of the biphoton state in the Fourier domain, a well-known property of broadband PDC (see, e.g., [4-6]), which in turn derives from the phase-matching conditions linking the emission angles and the frequencies of the emitted photons. The interdependence of correlated variables (known as multiparameter entanglement)

*enrico.brambilla@uninsubria.it has since long been recognized as a resource for engineering entanglement in quantum interferometric experiments [4,7], e.g., for controlling the spatial characteristics of the PDC light emitted in multimode waveguides [8,9]. In previous works [1-3], we demonstrated that under appropriate phase-matching conditions, the coupling between the spatial and the temporal frequencies translates in direct space into a linear relation between the temporal delays and the transverse separations of the twin photons at the crystal output face, within uncertainties in the femtosecond and in the micrometer range, respectively. This feature gives the PDC correlation function the characteristic X-shaped structure with skewed tails in the space-time domain (see, e.g., Fig. 3). The goal of the present work is a theoretical investigation of a scheme based on the use of sum frequency generation (SFG) to explore the predicted $\mathrm{X}$-shaped geometry of PDC entanglement. An experiment based on this scheme is currently under development at the Insubria University in Como [11,12].

At low gains, a prominent way to probe the twin photon correlation is the Hong-Ou- Mandel (HOM) detection scheme [13]. However, the experiment in Como works in the high-gain regime of $\mathrm{PDC}$, where the visibility of a HOM dip would be exceedingly low. We consider therefore an alternative detection scheme based on the SFG process taking place in a second nonlinear crystal, onto which the PDC source is imaged. This second crystal operates as an ultrafast optical correlator, the up-converted field containing the information about the correlation of the injected source.

Recent experimental works [14-18] used SFG to test the twin-photon or twin-beam correlation in the purely temporal domain, by imposing a controlled temporal delay between the twin photons. A careful theoretical modeling of such schemes, and of related schemes based on two-photon absorption [19], was developed in Ref. [18]. However, this analysis is restricted to models considering only the temporal degrees of freedom of light. A fully spatiotemporal model describing the upconversion of PDC photons via SFG has been considered in 
Ref. [17], however, this model is, in turn, valid only in the coincidence count regime of PDC.

The aim of the experiment being developed in Como is to use SFG to explore the PDC correlation in the whole spatiotemporal domain, by manipulating independently the temporal and the spatial degrees of freedom of the twin beams. To this end, we develop here a fully spatiotemporal model in order to describe the nonlinear processes taking place in the two crystals and the propagation between the crystals. This model is valid both in the high-gain regime of PDC, where the experiment in Como is being performed, and in the low-gain regime, where twin photons can be resolved by coincidence counting.

We demonstrate that the proposed scheme allows, in principle, the reconstruction of the X-shaped spatiotemporal correlation of twin beams or twin photons, and we identify the best conditions under which an experimental observation can be performed. In particular, we analyze important issues such as the visibility of the information thereby obtained (which becomes crucial in the high-gain regime of PDC) and the tolerance of the scheme with respect to common experimental imperfections, such as errors in the imaging scheme that maps the PDC light onto the SFG crystal or misalignments of the two nonlinear crystals.

Besides being necessary to describe the detection of the spatiotemporal $\mathrm{X}$ correlation, we remark that our fully spatiotemporal model for PDC and SFG is also crucial to interpret the results of similar schemes aimed at exploring the purely temporal correlation of twin photons, at least when the temporal bandwidths in play are large. This statement, further demonstrated by the experiment reported in Ref. [11], originates from the intrinsic nonfactorability of spatial and temporal degrees of freedom of the PDC correlation, which implies that the temporal properties of twin photons depend also on the way their spatial degrees of freedom are manipulated before being up-converted (by the use, e.g., of pupils that restrict the acceptance angle). A clear example is given in Sec. VIII b, where we show that the effect of spatial diffraction introduced by an imperfect imaging scheme induces a temporal broadening of the correlation, in addition to the more obvious spatial broadening.

The paper is organized as follows. Section II illustrates the proposed scheme, while in Sec. III we develop a modeling of the setup. In Sec. IV we derive a general solution for the coherence function of the generated SFG light, so that in Sec. V we are able to demonstrate how the full spatiotemporal $\mathrm{X}$ correlation can be retrieved by monitoring the SFG light intensity as a function of the temporal delay and the spatial transverse shift between the twin beams. Section VII discusses the issue of the visibility of the information, while Sec. VIII analyzes the tolerance of the scheme with respect to some common experimental imperfections.

\section{GENERAL DESCRIPTION OF THE SCHEME}

The main features regarding the proposed detection scheme and its theoretical modeling are the following.

(1) Both the spatial and the temporal degrees of freedom of the optical fields are taken into account. In order to observe $\mathrm{X}$ entanglement, a large temporal bandwidth (hundreds of

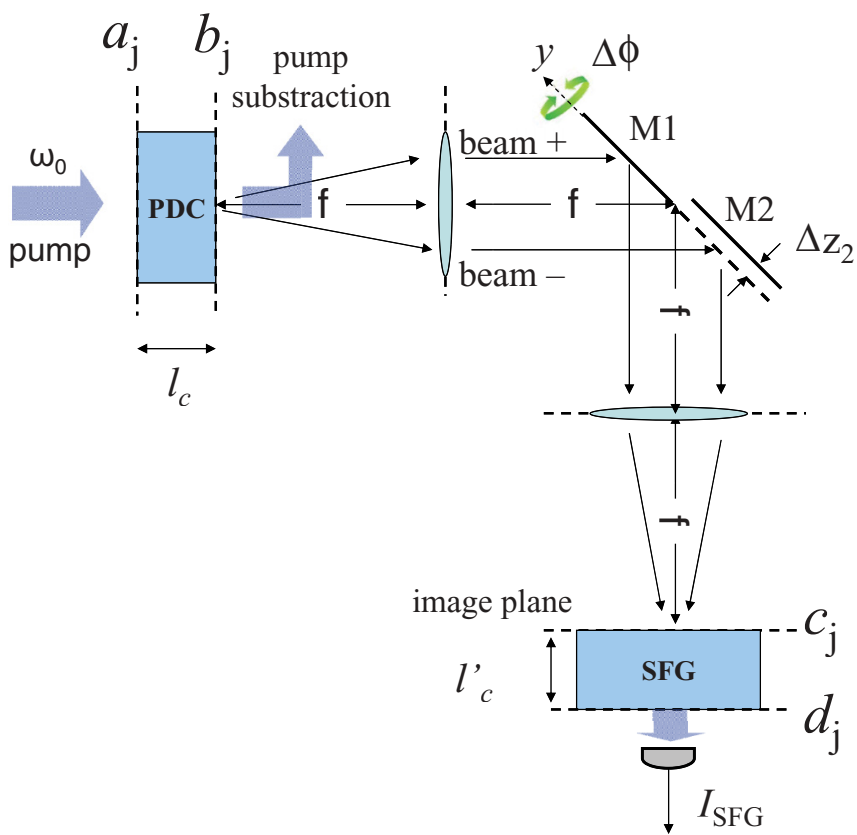

FIG. 1. (Color online) Proposed scheme for detecting the full spatiotemporal correlation of PDC via sum frequency generation. The PDC light generated by the first crystal is imaged onto the second, identical crystal, where up-conversion takes place. A transverse spatial displacement $\Delta x$ and a temporal delay $\Delta t$ are imposed independently on beam + and beam - , respectively, by means of rotations/translations of mirrors M1/M2 placed in the $2 f$ plane of the imaging system. The output SFG intensity is monitored as a a function of $\Delta x$ and $\Delta t$.

nanometers) of PDC radiation needs to be collected [1,2], so that special care will be taken to model broadband field propagation. The spatial bandwidth is also rather large so that we assume that optical elements have large acceptance angles.

(2) Our description is valid in any gain regime of PDC, so that it models both the generation and the detection of twin photons and of twin beams.

(3) A simplified scheme is illustrated in Fig. 1. The first nonlinear crystal, pumped by a broad coherent pump beam, generates PDC radiation. After eliminating the pump beam, the exit face of the PDC crystal is imaged onto the entrance face of the second nonlinear crystal, where the inverse SFG process takes place. This is described in Fig. 1 by a $4 f$ lens imaging system: in a real implementation, however, dispersive optical elements should be avoided as much as possible, since dispersion would drastically deteriorate the phase-sensitive correlation. The use of parabolic mirrors in place of lenses is a valid alternative, as we recently demonstrated in Refs. [10] and [11].

(4) In order to have an efficient up-conversion process, the SFG and PDC crystals must be of the same material and cut for the same phase-matching conditions, while their lengths can differ.

(5) We focus on type I PDC, and in the following we always refer to the case of a $\mathrm{BBO}$ ( $\beta$-barium-borate) crystal pumped at $527 \mathrm{~nm}$ in an e-oo phase-matching configuration. The PDC field is then described by a single field operator (ordinarily polarized), while the pump field is extraordinarily polarized. 
(6) In order to explore the full spatiotemporal structure of PDC entanglement, a key requirement is the ability to impose independently a temporal delay $\Delta t$ and a transverse spatial displacement $\Delta \vec{x}$ between the two twin components of PDC radiation. The twin components of the signal beams can be manipulated separately in the $2 f$ plane of the imaging device (the far-field plane with respect to both the PDC crystal exit face and the SFG crystal entrance face): the reason is that for a broad pump beam, twin photons are always emitted with opposite transverse wave vectors $\vec{q}$ and $-\vec{q}$, so that when a photon is found in the upper half of the $2 f$ plane, its twin will always be in the lower half-plane. We denote by beam + and beam -respectively, the upper and lower portions of the $2 f$ plane, which correspond to the two portions of the PDC radiation having positive $q_{y}>0$ and negative $q_{y}<0$ components of the transverse wave vector along the $y$ axis in Fig. 1.

(7) Since the PDC correlation is strongly localized both in space and in time (in the micrometer and femtosecond range, respectively [1-3]), the temporal and spatial relative displacements of the twin beams must be scanned with micrometric precision. This can be realized by means of the two plane mirrors M1 and M2 placed in the $2 f$ plane of the telescopic system. As described in detail in the following, a rotation of mirror M1 by an angle $\Delta \phi$ generates, at the SFG crystal input face, a transverse displacement of beam + of an amount $\Delta x=2 f \Delta \phi$, while a translation of mirror M2 by a distance $\Delta z_{2}$ generates a temporal delay $\Delta t=\Delta z_{2} / c$.

The intensity of the up-converted field generated by the SFG process in the second crystal is then monitored as a function of the relative temporal delay and spatial shift of the twin beams. The main idea that we demonstrate is that this quantity is able to give precise information about the structure of the correlation of twin beams generated in the first crystal and can be used in order to reconstruct the shape of this correlation in space and in time.

In the treatment that follows we develop a model that takes fully into account the propagation effects and the phase-matching mechanism that selects the spatiotemporal frequencies in both crystals.

\section{MODELING THE OPTICAL SYSTEM}

In order to describe the scheme in Fig. 1, we consider separately the propagation in the PDC crystal (Step I), the linear propagation between the two crystals (Step II), and the up-conversion process in the second crystal (Step III). As indicated in Fig. 1, the field operators in the different planes of interest are labeled with

(1) $a_{j}$ at the input plane of the PDC crystal,

(2) $b_{j}$ at the output plane of the PDC crystal,

(3) $c_{j}$ at the input plane of the SFG crystal, and

(4) $d_{j}$ at the output plane of the SFG crystal.

The $j=0$ index refers to beams with central frequency $\omega_{0}$ (either the pump field in the first crystal or the up-converted field in the second crystal), the $j=1$ index to the beams of central frequency $\omega_{1}=\omega_{0} / 2$ (either the down-converted field in the first crystal or the fundamental field in the SFG crystal).
The description of field evolution along $z$, the mean propagation axis of the system, is performed either in the direct spatiotemporal space $(\vec{x}, t)$, where $\vec{x} \equiv(x, y)$ is the (2D) transverse coordinate and $t$ is time, or in the Fourier spatiotemporal domain $(\vec{q}, \Omega)$, where $\vec{q}$ is the transverse component of the wave vector and $\Omega$ denotes the frequency offset from the central frequency.

For convenience, we use a compact notation for these spacetime coordinates by making the substitutions

$$
\begin{aligned}
(\vec{x}, t) & \rightarrow \vec{\xi}, \\
(\vec{q}, \Omega) & \rightarrow \vec{w}, \\
\vec{q} \cdot \vec{x}-i \Omega t & \rightarrow \vec{w} \cdot \vec{\xi} .
\end{aligned}
$$

Our analysis is carried out at two levels.

(1) In the limit where the pump beam driving the PDC process is broad and long enough, we adopt the plane-wave pump approximation (PWPA), which allows us to derive analytical or semianalytical results. This model is presented in Secs. III A-VII.

(2) In order to obtain results for a finite pump, we also developed a full 3D +1 numerical model, based on stochastic simulation of field evolutions. This model is introduced in Sec. VII.

\section{A. Step I: Propagation in the PDC crystal}

In this section we describe the model we use to describe PDC (also derived in Refs. [1], [6], and [2]), and we recall the main features of the space-time correlation of PDC light that we call $X$ entanglement [1-3].

The pump and down-converted fields are described by two field operators, $b_{0}$ and $b_{1}$, centered around the frequencies $\omega_{0}$ and $\omega_{1}=\omega_{0} / 2$, respectively. Normalization is such that $\left\langle b_{j}^{\dagger}(\xi) b_{j}(\xi)\right\rangle$ gives the photon number per unit area and unit time. The generation of the PDC field along the nonlinear crystal takes its simplest form in the Fourier domain

$$
b_{j}(\vec{w}, z)=\int \frac{d^{3} \xi}{(2 \pi)^{\frac{3}{2}}} b_{j}(\xi, z) e^{-i \xi \cdot \vec{w}}, \quad j=0,1,
$$

where we recall that $\vec{w}=(\vec{q}, \Omega)$ is the set of $3 \mathrm{D}$ Fourier coordinates, $\xi=(\vec{x}, t)$, while $z$ is the longitudinal coordinate along the mean propagation direction in the crystal. Next, we introduce the slowly varying amplitudes

$$
\begin{aligned}
& \bar{b}_{1}(\vec{w}, z)=e^{-i k_{1 z}(\vec{w}) z} b_{1}(\vec{w}, z), \\
& \bar{b}_{0}(\vec{w}, z)=e^{-i k_{0 z}(\vec{w}) z} b_{0}(\vec{w}, z),
\end{aligned}
$$

where $k_{j z}(\vec{w})=\sqrt{k_{j}(\vec{w})^{2}-q^{2}}$ is the $z$ component of the wave vector for the $j$ field. These amplitudes vary slowly along the $z$ coordinate, because their evolution is only due to the nonlinear interaction, since we have subtracted the effect of the fast linear propagation contained in the phase factor $\exp \left[i k_{j z}(\vec{w}) z\right]$. We can assume that the pump beam is undepleted by the PDC, so that its evolution is only linear, $\bar{b}_{0}(\vec{w}, z)=\bar{b}_{0}(\vec{w}, 0)$. In the same approximation, the pump operator can be substituted by its $c$-number amplitude $\bar{b}_{0}(\vec{w}, 0) \rightarrow \alpha_{p}(\vec{w})$. The propagation equation for the signal field contains only first-order $z$ 
derivatives and takes the form $[1,6]$

$$
\begin{aligned}
\frac{\partial}{\partial z} \bar{b}_{1}(\vec{w}, z)= & \sigma \int \frac{d \vec{w}^{\prime}}{(2 \pi)^{3 / 2}} \alpha_{p}\left(\vec{w}+\vec{w}^{\prime}\right) \bar{b}_{1}^{\dagger}\left(\vec{w}^{\prime}, z\right) \\
& \times e^{-i \Delta\left(\vec{w}, \vec{w}^{\prime}\right) z} .
\end{aligned}
$$

Here the phase-matching function

$$
\Delta\left(\vec{w}, \vec{w}^{\prime}\right)=k_{1 z}(\vec{w})+k_{1 z}\left(\vec{w}^{\prime}\right)-k_{0 z}\left(\vec{w}^{\prime}+\vec{w}^{\prime}\right)
$$

describes the phase mismatch between the two generated signal modes $(\vec{q}, \Omega),\left(\vec{q}^{\prime}, \Omega^{\prime}\right)$ and the pump mode $\left(\vec{q}+\vec{q}^{\prime}, \Omega+\Omega^{\prime}\right)$. Efficient down-conversion takes place only in those modes for which the phase mismatch is small. The coupling constant $\sigma$ is defined by

$$
\sigma=d_{\mathrm{eff}} \sqrt{\frac{\hbar \pi^{3} \omega_{0}^{3}}{4 \epsilon_{0} n_{0} n_{1}^{2} c^{3}}}
$$

where $d_{\text {eff }}$ is the effective second-order susceptibility of the nonlinear crystal, $n_{0}$ and $n_{1}$ are the refraction indexes at the central frequencies $\omega_{0}$ and $\omega_{1}$.

Equation (4) can be analytically solved in the plane-wave pump approximation, $\alpha_{p}\left(\vec{w}+\vec{w}^{\prime}\right) \rightarrow(2 \pi)^{3 / 2} \bar{\alpha}_{p} \delta\left(\vec{w}+\vec{w}^{\prime}\right)$, where $\bar{\alpha}_{p}$ denotes the pump field peak value in direct space. As analyzed in detail in Ref. [2], such an approximation holds as long as the pump beam waist and duration are larger than the spatial transverse displacement and temporal delay experienced by the pump and signal beams along the crystal because of walk-off and group velocity dispersion (in the example of a 4-mm-long BBO, a pump pulse with a waist larger than $\sim 250 \mu \mathrm{m}$ and a duration above $\sim 200 \mathrm{fs}$ satisfies this condition). The solution is expressed by a unitary transformation linking the field operators at the output face of the crystal, $b_{1}(\vec{w}) \equiv b_{1}\left(\vec{w}, z=l_{c}\right)$, to those at at the input face, $a_{1}(\vec{w}) \equiv b_{1}(\vec{w}, z=0)$ :

$$
b_{1}(\vec{w})=U(\vec{w}) a_{1}(\vec{w})+V(\vec{w}) a_{1}^{\dagger}(-\vec{w})
$$

The explicit expression of the functions $U$ and $V$ can be found, e.g., in Ref. [2]. Here we note that they depend on $\vec{w}$ only through the plane-wave pump phase-matching function

$$
\Delta^{\mathrm{pdc}}(\vec{w}) \equiv \Delta(\vec{w},-\vec{w})=k_{1 z}(\vec{w})+k_{1 z}(-\vec{w})-k_{0},
$$

where $k_{0}=n_{0} \omega_{0} / c$ is the wave number of the pump.

All the properties of the PDC light are described by the following second-order field correlation functions:

$$
\begin{aligned}
\left\langle b_{1}^{\dagger}(\vec{w}) b_{1}\left(\vec{w}^{\prime}\right)\right\rangle & =\delta\left(\vec{w}-\vec{w}^{\prime}\right)|V(\vec{w})|^{2}, \\
\left\langle b_{1}(\vec{w}) b_{1}\left(\vec{w}^{\prime}\right)\right\rangle & =\delta\left(\vec{w}+\vec{w}^{\prime}\right) U(\vec{w}) V(-\vec{w}) .
\end{aligned}
$$

In particular, from Eq. (9b), we see that the function

$$
F_{\mathrm{PDC}}(\vec{w}) \equiv U(\vec{w}) V(-\vec{w})
$$

represents the probability amplitude that a pump photon at $(\vec{q}=0, \Omega=0)$ is down-converted into a pair of phaseconjugated photons $(\vec{q}, \Omega)$ and $(-\vec{q},-\Omega)$. Its explicit expression is

$$
\begin{aligned}
F_{\mathrm{PDC}}(\vec{w}) & =g e^{i k_{0} l_{c}} \frac{\sinh \Gamma(\vec{w}) l_{c}}{\Gamma(\vec{w}) l_{c}}\left\{\cosh \Gamma(\vec{w}) l_{c}\right. \\
& \left.+i \frac{\Delta^{\mathrm{pdc}}(\vec{w}) l_{c}}{2 \Gamma(\vec{w}) l_{c}} \sinh \Gamma(\vec{w}) l_{c}\right\},
\end{aligned}
$$

$$
\Gamma(\vec{w}) l_{c}=\sqrt{g^{2}-\frac{\left[\Delta^{\mathrm{pdc}}(\vec{w}) l_{c}\right]^{2}}{4}},
$$

where $g$ is the dimensionless gain parameter proportional to the pump peak amplitude,

$$
g=\sigma l_{c} \bar{\alpha}_{p}
$$

The other relevant function is the PDC spatiotemporal spectrum

$$
|V(\vec{w})|^{2}=g^{2} \frac{\sinh ^{2}\left[\Gamma(\vec{w}) l_{c}\right]}{\Gamma^{2}(\vec{w}) l_{c}^{2}},
$$

which gives the photon number distribution in the spatiotemporal Fourier domain.

We remark that Eqs. (4)-(13) are valid in any gain regime of PDC. In the low-gain regime $g \ll 1$ they describe the downconversion of pump photons into pairs of signal-idler photons that can be resolved individually. In the high-gain regime $g \sim$ 1 stimulated down-conversion becomes important, and these equations describe the generation of macroscopic twin beams of light made of "bunched" pairs of twin photons.

In particular, in the low-gain regime Eq. (11) takes the well-known Sinc dependence on the phase mismatch:

$$
\lim _{g \ll 1} F_{\mathrm{PDC}}(\vec{w})=g e^{i k_{0} l_{c}} \operatorname{Sinc} \frac{\Delta^{\mathrm{pdc}}(\vec{w}) l_{c}}{2} e^{i \frac{\Delta^{\mathrm{pdc}}(\vec{w}) l_{c}}{2}} .
$$

In Ref. [1] the term X entanglement was used to describe the shape of the spatiotemporal correlation of the biphoton amplitude at the crystal output face. The quantity of interest is therefore

$$
\psi_{\mathrm{PDC}}(\vec{\xi})=\left\langle b_{1}(\vec{\xi}) b_{1}(\vec{\xi}+\Delta \vec{\xi})\right\rangle
$$

which, in the stationary plane-wave pump regime, depends only on the relative spatial and temporal coordinates $\Delta \xi \equiv$ $(\Delta \vec{x}, \Delta t)$. It can be expressed [2] as the Fourier transform of the spectral probability amplitude $F_{\mathrm{PDC}}(\vec{w})$ of generating photons in phase conjugate modes $\vec{w}$ and $-\vec{w}$, that is,

$$
\psi_{\mathrm{PDC}}(\xi)=\int \frac{d \vec{w}}{(2 \pi)^{3}} e^{i \vec{\xi} \cdot \vec{w}} F_{\mathrm{PDC}}(\vec{w}) .
$$

Figure 2 shows the behavior of $\left|F_{\mathrm{PDC}}\right|$ for collinear phase matching, calculated by using the Sellmaier relations for the refractive indexes [22]. As can be inferred from Eqs. (11) and $(14)$, in any gain regime $F_{\mathrm{PDC}}(\vec{w})$ is strongly peaked around the phase-matching curves defined by $\Delta^{\mathrm{pdc}}(\vec{w})=0$. The hyperbolic geometry displayed in the neighborhood of degeneracy can be understood by making a quadratic expansion of $k_{z}$ around $(\vec{q}=0, \Omega=0)$,

$$
k_{1 z}(\vec{q}, \Omega) \approx k_{1}+k_{1}^{\prime} \Omega+\frac{1}{2} k_{1}^{\prime \prime} \Omega^{2}-\frac{q^{2}}{2 k_{1}},
$$

where $\quad k_{1}=k_{1}(\vec{w}=0), \quad k_{1}^{\prime}=d k_{1} /\left.d \Omega\right|_{\vec{w}=0}, \quad$ and $k_{1}^{\prime \prime}=d^{2} k_{j} /\left.d \Omega^{2}\right|_{\vec{w}=0} \cdot{ }^{1}$ The phase-matching function, (8),

\footnotetext{
${ }^{1} \mathrm{~A}$ detailed discussion on the range of validity of approximations (17) can be found in Ref. [3] in the context of type II phase matching.
} 


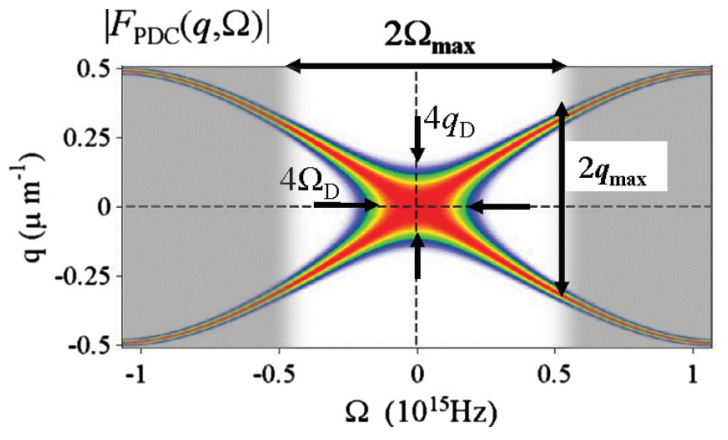

FIG. 2. (Color online) Plot of $\left|F_{\mathrm{PDC}}\right|$ in the $\left(q_{x}, \Omega\right)$ plane, for a 4-mm-long type I BBO pumped at $527 \mathrm{~nm}$, cut for collinear phase matching. The unshaded region corresponds to the frequency filter used in the simulation with $2 \Omega_{\max }=0.965 \times 10^{15} \mathrm{~Hz}$ FWHM. The bandwidths $\sim 4 q_{D}$ and $\sim 4 \Omega$ of the phase matching of $\left|F_{\mathrm{PDC}}\right|$ along the spatial and the temporal frequency axis are indicated. The parametric gain is $g=8, \Delta_{0}^{\mathrm{PDC}}=0$.

then takes the quadratic form

$$
\Delta^{\mathrm{pdc}}(\vec{w}) l_{c} \approx \Delta_{0}^{\mathrm{PDC}} l_{c}+\frac{q^{2}}{q_{D}^{2}}-\frac{\Omega^{2}}{\Omega_{D}^{2}},
$$

where $\Delta_{0}^{\mathrm{PDC}}=2 k_{1}-k_{0}$ is the collinear phase-mismatch parameter, and

$$
\Omega_{D}=\sqrt{1 / k_{1}^{\prime \prime} l_{c}}, \quad q_{D}=\sqrt{k_{1} / l_{c}} .
$$

$q_{D}$ and $\Omega_{D}$ determine the characteristic scale of variations of $F_{\mathrm{PDC}}$ along the $\Omega$ axis, at fixed $q$, and along the $q$ axis at fixed $\Omega$, respectively. They scale with the inverse square root of the crystal length and are generally much smaller than the range of frequencies of the whole PDC emission spectrum (the latter can, in principle, extend up to the pump optical frequency). When tuning the crystal for collinear phase matching, i.e., for $\Delta_{0}^{\mathrm{PDC}}=0$, phase matching occurs along the lines $q / q_{D}=$ $\pm \Omega / \Omega_{D}$. Under this condition, we note that the first zeros of the function $\Gamma(\vec{q}, \Omega) l_{c}$ [see Eq. (11b)] along the $\Omega$ and $q$ axis, evaluated using approximation (18),

$$
\bar{\Omega}_{D}=\sqrt{2}\left(\pi^{2}+g^{2}\right)^{1 / 4} \Omega_{D}, \quad \bar{q}_{D}=\sqrt{2}\left(\pi^{2}+g^{2}\right)^{1 / 4} q_{D},
$$

provide good estimates of the widths of $\left|F_{\mathrm{PDC}}\right|$ along those axis, which take into account the gain broadening effect in the spectral domain. For the example shown in Fig. 2, with $g=8$, we have $\bar{\Omega}_{D} \approx 4 \Omega_{D}, \bar{q}_{D} \approx 4 q_{D}$, as indicated by the arrows.

The hyperbolic geometry of phase matching in the spectral domain turns into a characteristic $\mathrm{X}$-shaped geometry for the spatiotemporal biphoton correlation $\psi_{\mathrm{PDC}}(\Delta \vec{x}, \Delta t)$, (16). Figure 3(a) shows the profile of $\psi_{\mathrm{PDC}}$ as a function of the relative spatial and temporal coordinates. (Only one transverse dimension is shown.) The tails of the structure are oriented along the lines $\Omega_{D} \Delta t= \pm q_{D} \Delta x$, a feature expressing a linear relation between the temporal delay and the spatial transverse separation acquired by the twin photons when arriving at the crystal output face [1,2], i.e., making use of Eqs. (19),

$$
\Delta t= \pm \sqrt{k_{1}^{\prime \prime} k_{1}} \Delta x
$$

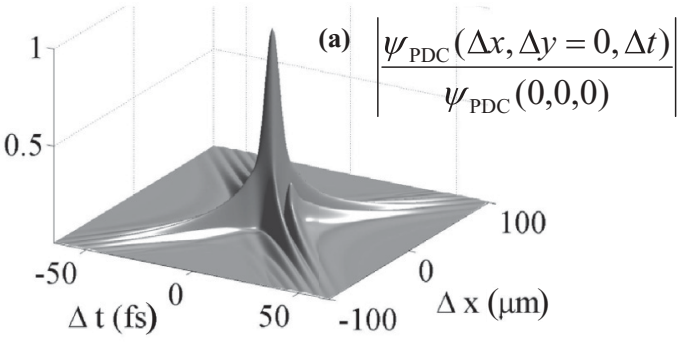
(b) $\left|\frac{\psi_{\mathrm{PDC}}(\Delta \vec{x}=0, \Delta t)}{\psi_{\mathrm{PDC}}(0,0)}\right|^{2}$
(c) $\left|\frac{\psi_{\mathrm{PDC}}(\Delta x, \Delta y=0, \Delta t=0)}{\psi_{\mathrm{PDC}}(0,0,0)}\right|^{2}$
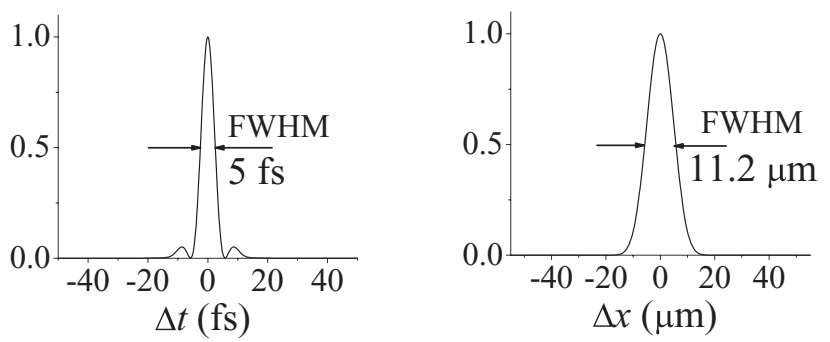

FIG. 3. Example of $X$ entanglement for the same crystal as in Fig. 2. (a) Plot of the modulus of the biphoton amplitude as a function of the relative spatiotemporal coordinates $\Delta x$ and $\Delta t$. (b) Temporal and (c) spatial profiles of the central peak of the correlation. Same parameters as in Fig. 2.

As described in detail in Ref. [2], when tilting the crystal away from collinear phase matching, this linear relation becomes less and less stringent: as a consequence, the tails of the structure become progressively less visible. The $\Delta_{0}^{\mathrm{PDC}}=0$ phase-matching condition therefore represents the optimal configuration for observing $\mathrm{X}$ entanglement.

A relevant feature of $\mathrm{X}$ entanglement is the strong localization of the central peak of the correlation function, whose cross sections along the temporal and the spatial axes are shown in the lower part in Fig. 3. The width of the peak is, in principle, determined by the inverse of the full PDC emission bandwidth or, in practice, by the bandwidth intercepted in the measurement. In the example in the figure, we simulated the presence of a filter in the temporal frequency of width $2 \Delta \Omega_{\max }=0.965 \times 10^{15} \mathrm{~Hz}$ (indicated by the unshaded region in Fig. 2), which corresponds to a wavelength interval ranging from $830 \mathrm{~nm}$ up to the conjugate wavelength $1444 \mathrm{~nm}$. The temporal profile of the correlation in Fig. 3 has a full width at half-maximum $\Delta t_{\mathrm{fwhm}} \approx 5 \mathrm{fs}$, close to that of the Fourier transform of a box function of width $2 \Omega_{\max }$. The spatial width of the correlation peak, in turn, is determined by the range of spatial frequencies involved in the PDC emission and, typically, is of the order of a few micrometers.

\section{B. Step II: Propagation between the two crystals}

We assume ideally that the optical setup illustrated in Fig. 1 behaves as a perfect imaging system, free of dispersion and losses. In the absence of any temporal delay and spatial shift $(\Delta t=0, \Delta x=0)$, the PDC field at the PDC crystal exit face is then mapped into the SFG crystal entrance face $c_{1}(\vec{x}, t)=$ 
$b_{1}(\vec{x}, t) .^{2}$ In order to model the effect of a small rotation of mirror M1 and a small translation of mirror M2, we assume that the propagation angles in play are such that the paraxial approximation holds.

Let us first consider the temporal delay applied to the $q_{y}<0$ Fourier modes of the PDC light (beam -). By translating mirror M2 by $\Delta z_{2}$ the separation between the two lenses in Fig. 1 becomes $2 f+\Delta z_{2}$. The fields at frequency $\omega_{1}+\Omega$ in the left and right focal planes of the imaging device are related through the algebraic transformation (the $\vec{x} \rightarrow-\vec{x}$ reflection is omitted)

$$
c_{1}(\vec{x}, \Omega)=e^{i \frac{\omega_{1}+\Omega}{c}\left(1-\frac{|\vec{x}|^{2}}{f^{2}}\right) \Delta z_{2}} b_{1}(\vec{x}, \Omega) .
$$

The effect of diffraction due to the additional propagation $\Delta z_{2}$ is described, within the paraxial approximation, by the quadratic phase factor proportional to $|\vec{x}|^{2} / f^{2}$. Its effect can be neglected as long as

$$
\Delta z \ll \frac{\lambda}{\pi} \frac{f^{2}}{|\vec{x}|^{2}} .
$$

Referring to the conditions of the experiment [10,11], for $f \sim 20 \mathrm{~cm}, \lambda \sim 1 \mu \mathrm{m}$, and $|\vec{x}| \sim w_{p} \leqslant 1 \mathrm{~mm}$, the condition reads $\Delta z \ll 1.2 \mathrm{~cm}$. In order to explore the X-shaped PDC correlation, delays of a few hundred femtoseconds at most are sufficient [see, e.g., the plot in Fig. 3(a)], so that, in practice, condition (23) is always fulfilled. Each Fourier mode of beam - therefore undergoes, within a very good approximation, the diffractionless transformation

$$
c_{1}(\vec{q}, \Omega)=e^{i \frac{\Omega}{c} \Delta z_{2}} b_{1}(\vec{q}, \Omega) \quad \text { for } \quad q_{y}<0,
$$

where an inessential constant phase factor has been omitted.

We now consider the manipulation of beam + through the rotating mirror M1. As shown in the Appendix, a rotation of M1 by a small angle $\Delta \phi$ around a given axis generates a transverse shift $\Delta s=2 f \Delta \phi$ of the beam at the entrance face of the SFG crystal (the imaging plane), in the direction orthogonal to the rotation axis. According to Eq. (A6), the complete transformation undergone by beam + between the input and the output planes of the $4 f$ telescopic system can be written in the Fourier domain in the form (the $\vec{q} \rightarrow-\vec{q}$ reflection and the minus sign are omitted here for simplicity)

$$
c_{1}(\vec{q}, \Omega)=e^{i \vec{q} \cdot \Delta \vec{s}} b_{1}(\vec{q}, \Omega) \text { for } \quad q_{y}>0,
$$

where $\Delta \vec{s}$ denotes the transverse shift generated by a rotation of mirror M1 around a generic axis. Putting together relations (24) and (25), the overall transformation describing propagation from the PDC crystal output face to the SFG crystal input face can therefore be synthesized into the unitary input-output relation

$$
c_{1}(\vec{q}, \Omega)=\left[\Theta\left(q_{y}\right) H_{+}(\vec{q}, \Omega)+\Theta\left(-q_{y}\right) H_{-}(\vec{q}, \Omega)\right] b_{1}(\vec{q}, \Omega),
$$

\footnotetext{
${ }^{2}$ For simplicity, we neglect here and in the following the spatial reflection with respect to the $z$ axis $\vec{x} \rightarrow-\vec{x}$, as well as constant phase factors.
}
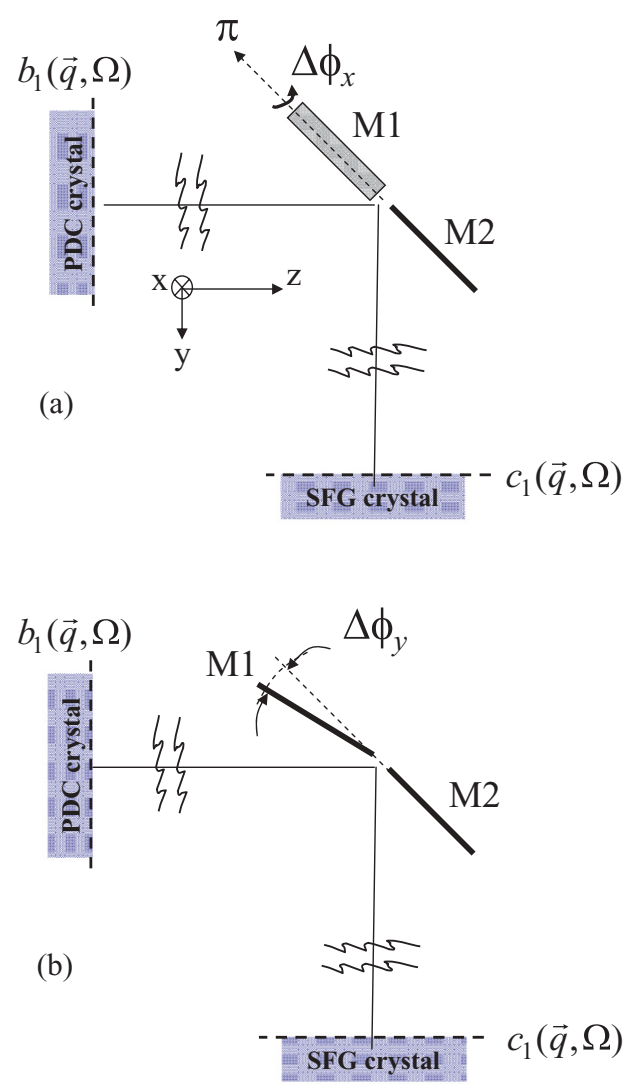

FIG. 4. (Color online) In configuration (a) the rotation by a small angle $\Delta \phi_{x}$ of mirror M1 around the $\pi$ axis produces the displacement $\Delta \vec{s}=\left(\Delta x=2 f \Delta \phi_{x}, 0\right)$ along the $x$ direction at the entrance face of the SFG crystal (orthogonal to the figure plane). In case (b) the rotation is performed around the $x$ axis and produces the displacement $\Delta \vec{s}=\left(0, \Delta y=2 f \Delta \phi_{y}\right)$ along the $y$ direction.

where $\Theta\left(q_{y}\right)$ denotes the step function, equal to 1 for $q_{y} \geqslant 0$ and to 0 for $q_{y}<0$, and

$$
H_{+}(\vec{q}, \Omega)=e^{i \vec{q} \cdot \Delta \vec{s}}, \quad H_{-}(\vec{q}, \Omega)=e^{i \frac{\Omega}{c} \Delta z_{2}}
$$

are the transfer functions associated with beam + and beam - , respectively. The identity $\Theta\left(q_{y}\right)+\Theta\left(-q_{y}\right) \equiv 1$ guarantees that the commutation rules are preserved by the transformation.

It is important to note that the correlation measurement depends on the particular choice of the rotation axis of M1, as shown in Sec.V. We consider explicitly the two configurations illustrated in Fig. 4: in case (a) M1 is rotated around the $\pi$ axis orthogonal to the gap between the two mirrors, while in case (b) the rotation axis $\pi$ of M1 is coincident with the $x$ axis (orthogonal to the figure plane). We thus have

$$
\begin{aligned}
& \Delta \vec{s}=(\Delta x, 0), \quad \Delta x=2 f \Delta \phi_{x} \quad \text { in case (a), } \\
& \Delta \vec{s}=(0, \Delta y), \quad \Delta y=2 f \Delta \phi_{y} \quad \text { in case (b), }
\end{aligned}
$$

where $\Delta \phi_{x}$ and $\Delta \phi_{y}$ denote a small rotation angle applied to the mirror around the $\pi$ axis in configuration (a) and around the $x$ axis in configuration (b).

\section{Step III: Propagation in the SFG crystal}

We now model the generation of the up-converted field inside the SFG crystal. This process is just the reverse of the 
down-conversion described in Sec. III A. Following the same procedure outlined there, we work in the Fourier domain and we introduce the slowly varying amplitudes of the fundamental $d_{1}$ (carrier frequency $\omega_{1}$ ) and second harmonic $d_{0}$ (carrier frequency $\omega_{0}$ ) fields along the SFG crystal

$$
\begin{aligned}
& \bar{d}_{0}(\vec{w}, z)=e^{-i k_{0 z}(\vec{w}) z} d_{0}(\vec{w}, z), \\
& \bar{d}_{1}(\vec{w}, z)=e^{-i k_{1 z}(\vec{w}) z} d_{1}(\vec{w}, z),
\end{aligned}
$$

which vary along $z$ only because of the nonlinear interaction, since we have subtracted the effect of linear propagation. Their evolution is described by the following pair of coupled equations:

$$
\frac{\partial \bar{d}_{0}(\vec{w}, z)}{\partial z}=-\sigma \int \frac{d \vec{w}^{\prime}}{(2 \pi)^{3 / 2}} \bar{d}_{1}\left(\vec{w}^{\prime}, z\right) \bar{d}_{1}\left(\vec{w}-\vec{w}^{\prime}, z\right) e^{i \Delta\left(\vec{w}{ }^{\prime}, \vec{w}-\vec{w}^{\prime}\right) z},
$$

$$
\frac{\partial \bar{d}_{1}(\vec{w}, z)}{\partial z}=\sigma \int \frac{d \vec{w}^{\prime}}{(2 \pi)^{3 / 2}} \bar{d}_{0}\left(\vec{w}+\vec{w}^{\prime}, z\right) \bar{d}_{1}^{\dagger}\left(\vec{w}^{\prime}, z\right) e^{-i \Delta\left(\vec{w}, \vec{w}^{\prime}\right) z}
$$

Equation (30a) describes all the up-conversion processes where a pair of fundamental photons in modes $\vec{w}^{\prime}, \vec{w}-\vec{w}^{\prime}$ are up-converted into a SFG photon in mode $\vec{w}$. Accordingly, in this equation $\Delta\left(\vec{w}^{\prime}, \vec{w}-\vec{w}^{\prime}\right)=k_{1 z}\left(\vec{w}^{\prime}\right)+k_{1 z}\left(\vec{w}-\vec{w}^{\prime}\right)-$ $k_{0 z}(\vec{w})$ represents the phase mismatch of such a process in the second SFG crystal. Equation (30b) is obviously just the same as Eq. (4) describing the down-conversion process.

Equations (30) need to be considered together with the initial conditions at the crystal input face. For the fundamental field we have

$$
\bar{d}_{1}(\vec{w}, z=0)=c_{1}(\vec{w})
$$

where $c_{1}$ is the field down-converted in the first PDC crystal, after steps I and II. For the second harmonic field, we assume that the pump field is completely eliminated after the PDC crystal, so that it is in the vacuum state at the SFG crystal input.

We now assume that the SFG crystal is short enough so that only a small fraction of the PDC light is up-converted. Under these conditions, the fundamental field remains basically unchanged during propagation, and Eq. (30) can be solved with a perturbative approach similar to that used in the low-gain regime of PDC in Ref. [2] (see Appendix therein). In this way, we obtain an explicit expression that links the operators at the SFG crystal output plane to those at the SFG input plane:

$$
\begin{aligned}
d_{1}(\vec{w}) \equiv & d_{1}\left(\vec{w}, l_{c}^{\prime}\right)=e^{i k_{1 z}(\vec{w}) l_{c}^{\prime}} c_{1}(\vec{w}) \\
d_{0}(\vec{w}) \equiv & d_{0}\left(\vec{w}, l_{c}^{\prime}\right)=e^{i k_{0 z}(\vec{w}) l_{c}^{\prime}}\left[c_{0}(\vec{w})-\int \frac{d \vec{w}^{\prime}}{(2 \pi)^{3 / 2}} c_{1}\left(\vec{w}-\vec{w}^{\prime}\right)\right. \\
& \left.\times c_{1}\left(\vec{w}^{\prime}\right) F_{\mathrm{SFG}}\left(\vec{w}-\vec{w}^{\prime}, \vec{w}^{\prime}\right)\right]
\end{aligned}
$$

where

$$
F_{\mathrm{SFG}}\left(\vec{w}, \vec{w}^{\prime}\right)=\sigma l_{c}^{\prime} e^{i \frac{\Delta\left(\vec{w}, \vec{w}^{\prime}\right) l_{c}^{\prime}}{2}} \operatorname{Sinc} \frac{\Delta\left(\vec{w}, \vec{w}^{\prime}\right) l_{c}^{\prime}}{2}
$$

The function $F_{\mathrm{SFG}}\left(\vec{w}, \vec{w}^{\prime}\right)$ can be interpreted as the probability amplitude density ${ }^{3}$ that a pair of photons in the fundamental modes $\vec{w} \equiv(\vec{q}, \Omega)$ and $\vec{w}^{\prime} \equiv\left(\vec{q}^{\prime}, \Omega^{\prime}\right)$ is up-converted into the second-harmonic mode $\vec{w}+\vec{w}^{\prime} \equiv\left(\vec{q}+\vec{q}^{\prime}, \Omega+\Omega^{\prime}\right)$ : this up-conversion probability is non-negligible only for those pairs of modes for which the phase mismatch $\Delta\left(\vec{w}, \vec{w}^{\prime}\right) l_{c}^{\prime} \approx 0$. As can be expected, the probability amplitude for such a process is formally identical to that of the reverse process of down-conversion. Equation (33) indeed has the same form as Eq. (14), the main difference being that Eq. (14) describes PDC only in the plane-wave pump limit, where the only allowed down-conversion processes are those leading to twin photons in modes $\vec{w}$ and $\vec{w}^{\prime}=-\vec{w}$.

\section{GENERAL SOLUTION: THE SFG COHERENCE FUNCTION}

We now put together the chain of field transformations presented in the previous section. Our main goal is to evaluate the intensity of the SFG field, but we start from a more general result, i.e., the coherence function of the SFG field in the spectral domain, evaluated at the output face of the SFG crystal,

$$
\mathcal{C}_{\mathrm{SFG}}\left(\vec{w}_{0}, \vec{w}_{0}^{\prime}\right)=\left\langle d_{0}^{\dagger}\left(\vec{w}_{0}\right) d_{0}\left(\vec{w}_{0}^{\prime}\right)\right\rangle
$$

Inserting the input output relation, (32b), for the SFG crystal inside this expression, we obtain an equation that links the SFG coherence function to the correlation functions of the fundamental field $c_{1}$ at the crystal input face. These correlations can be calculated by using relations (9), together with the identities $\left|H_{+}(\vec{w})\right|^{2}=\left|H_{-}(\vec{w})\right|^{2} \equiv 1$ and $\Theta\left(q_{y}\right)+\Theta\left(-q_{y}\right) \equiv$ 1. We obtain the following expressions:

$$
\begin{aligned}
\left\langle c_{1}^{\dagger}(\vec{w}) c_{1}\left(\vec{w}^{\prime}\right)\right\rangle= & \delta\left(\vec{w}-\vec{w}^{\prime}\right)|V(\vec{w})|^{2}, \\
\left\langle c_{1}(\vec{w}) c_{1}\left(\vec{w}^{\prime}\right)\right\rangle= & \delta\left(\vec{w}+\vec{w}^{\prime}\right) U(\vec{w}) V(-\vec{w}) \\
& \times\left[\Theta\left(q_{y}\right) H_{+}(\vec{w}) H_{-}(-\vec{w})\right. \\
& \left.+\Theta\left(-q_{y}\right) H_{+}(-\vec{w}) H_{-}(\vec{w})\right] .
\end{aligned}
$$

Using these relations we obtain, after some manipulations, the result

$$
\mathcal{C}_{\mathrm{SFG}}\left(\vec{w}_{0}, \vec{w}_{0}^{\prime}\right)=\mathcal{C}_{\mathrm{SFG}}^{(\mathrm{inc})}\left(\vec{w}_{0}, \vec{w}_{0}^{\prime}\right)+\mathcal{C}_{\mathrm{SFG}}^{(\mathrm{coh})}\left(\vec{w}_{0}, \vec{w}_{0}^{\prime}\right),
$$

with

$$
\begin{aligned}
\mathcal{C}_{\mathrm{SFG}}^{(\mathrm{inc})}\left(\vec{w}_{0}, \vec{w}_{0}^{\prime}\right)= & 2 \delta\left(\vec{w}_{0}-\vec{w}_{0}^{\prime}\right) \int \frac{d \vec{w}}{(2 \pi)^{3}}\left|V\left(\vec{w}_{0}-\vec{w}\right)\right|^{2} \\
& \times|V(\vec{w})|^{2}\left|F_{\mathrm{SFG}}\left(\vec{w}_{0}-\vec{w}, \vec{w}\right)\right|^{2}, \\
\mathcal{C}_{\mathrm{SFG}}^{(\mathrm{coh})}\left(\vec{w}_{0}, \vec{w}_{0}^{\prime}\right)= & \delta\left(\vec{w}_{0}\right) \delta\left(\vec{w}_{0}^{\prime}\right) \mid 2 \int \frac{d \vec{w}}{(2 \pi)^{3 / 2}} \Theta\left(q_{y}\right) H_{+}(\vec{w}) \\
& \times H_{-}(-\vec{w}) F_{\mathrm{PDC}}(\vec{w}) F_{\mathrm{SFG}}(\vec{w},-\vec{w}),\left.\right|^{2}
\end{aligned}
$$

where $F_{\mathrm{PDC}}(\vec{w})$ and $F_{\mathrm{SFG}}\left(\vec{w}, \vec{w}^{\prime}\right)$ are the spectral probability amplitudes defined in Eqs. (11) and (33).

\footnotetext{
${ }^{3}$ Its square modulus $\left|F_{\mathrm{SFG}}\left(\vec{w}, \vec{w}^{\prime}\right)\right|^{2}$ gives the probability per unit of spectral bandwidth.
} 
The incoherent term, (37a), originates from the upconversion of PDC photons which do not belong to phaseconjugated mode pairs. By contrast, the coherent term, (37b), originates from the up-conversion of photon pairs coming from phase-conjugate PDC modes, the latter process leading to the partial reconstruction of the original coherent pump beam as described in the experiment in Ref. [10]. In this regard, it is convenient to introduce a special symbol for the up-conversion probability amplitude appearing in Eq. (37b), namely,

$$
\begin{aligned}
F_{\mathrm{SFG}}^{(\mathrm{coh})}(\vec{w}) & \equiv F_{\mathrm{SFG}}(\vec{w} ;-\vec{w}) \\
& =\sigma l_{c}^{\prime} e^{i \frac{\Delta^{\mathrm{sfg}}(\vec{w}) l_{c}^{\prime}}{2}} \operatorname{Sinc} \frac{\Delta^{\mathrm{sfg}}(\vec{w}) l_{c}^{\prime}}{2}
\end{aligned}
$$

where

$$
\Delta^{\mathrm{sfg}}(\vec{w}) \equiv \Delta(\vec{w},-\vec{w})=k_{1}(\vec{w})+k_{1}(-\vec{w})-k_{0}^{\mathrm{sfg}}
$$

is the phase mismatch function for these coherent upconversion processes. The function $F_{\mathrm{SFG}}^{(\mathrm{coh})}$ plays a fundamental role in determining the properties of the coherent component of the SFG field. It describes the efficiency with which photons belonging to a particular pair of phase-conjugated modes $\vec{w}$ and $-\vec{w}$ undergoes the inverse process of the original PDC event, i.e., the back-conversion into the monochromatic plane-wave mode $(\vec{w}=0)$ corresponding to the original pump driving the PDC. The corresponding phase mismatch $\Delta^{\text {sfg }}$ is identical to the PDC phase-matching function, (8), except for the presence of the wave number $k_{0}^{\mathrm{sfg}}$, which differs from the pump wave number $k_{0}$ only in the case when the two crystals are not perfectly aligned (for the type I e-oo phase matching we are considering, only the pump beam $k$ vector depends on direction). When the two crystals are aligned, $F_{\mathrm{SFG}}^{(\mathrm{coh})}$ coincides with the probability amplitude for the reverse PDC process in the low-gain regime, as given by Eq. (14) (apart from a constant factor).

As can be inferred by comparing Eqs. (37b) and (16), only the coherent contribution contains the information about the biphoton correlation $\psi_{\text {PDC }}(\vec{\xi})$ we are looking for. The incoherent contribution rather acts as a background which tends to deteriorate the visibility of the correlation measurement [18].

Note also that the coherent component, (37b), factorizes into the product of the SFG field mean values,

$$
\mathcal{C}_{\mathrm{SFG}}^{(\mathrm{coh})}\left(\vec{w}, \vec{w}^{\prime}\right)=\left\langle d_{0}^{\dagger}(\vec{w})\right\rangle\left\langle d_{0}\left(\vec{w}^{\prime}\right)\right\rangle
$$

with

$$
\begin{aligned}
\left\langle d_{0}(\vec{w})\right\rangle= & \delta(\vec{w}) \int \frac{d \vec{w}^{\prime}}{(2 \pi)^{3 / 2}} H_{+}\left(\vec{w}^{\prime}\right) \\
& \times H_{-}\left(-\vec{w}^{\prime}\right) F_{\mathrm{PDC}}\left(\vec{w}^{\prime}\right) F_{\mathrm{SFG}}^{(\mathrm{coh})}\left(\vec{w}^{\prime}\right) .
\end{aligned}
$$

From Eqs. (36) and (37) we can evaluate the SFG intensity (number of photons per unit area and unit time) at the exit face of the second crystal, obtaining

$$
I_{\mathrm{SFG}}(\vec{\xi}) \equiv\left\langle d_{0}^{\dagger}(\vec{\xi}) d_{0}(\vec{\xi})\right\rangle=I_{\mathrm{SFG}}^{(\mathrm{coh})}+I_{\mathrm{SFG}}^{(\mathrm{inc})}
$$

with

$$
\begin{aligned}
I_{\mathrm{SFG}}^{(\mathrm{inc})}= & 2 \int \frac{d \vec{w}}{(2 \pi)^{3}} \int \frac{d \vec{w}^{\prime}}{(2 \pi)^{3}}|V(\vec{w})|^{2}\left|V\left(\vec{w}^{\prime}\right)\right|^{2}\left|F_{\mathrm{SFG}}\left(\vec{w}, \vec{w}^{\prime}\right)\right|^{2} \\
I_{\mathrm{SFG}}^{(\mathrm{coh})}= & \mid 2 \int \frac{d \vec{w}^{\prime}}{(2 \pi)^{3}} \Theta\left(q_{y}\right) H_{+}\left(\vec{w}^{\prime}\right) H_{-}\left(-\vec{w}^{\prime}\right) F_{\mathrm{PDC}}\left(\vec{w}^{\prime}\right) \\
& \times\left. F_{\mathrm{SFG}}^{(\mathrm{coh})}\left(\vec{w}^{\prime}\right)\right|^{2}
\end{aligned}
$$

The intensity distribution of the SFG light in the near field is uniform in space and time, an artifact due to the spatiotemporal invariance of our model deriving from the monochromatic and plane-wave pump approximation. By comparing Eqs. (37b) and (43b), we obtain the following relevant identity,

$$
\mathcal{C}_{\mathrm{SFG}}^{(\mathrm{coh})}\left(\vec{w}, \vec{w}^{\prime}\right)=(2 \pi)^{3} \delta(\vec{w}) \delta\left(\vec{w}^{\prime}\right) I_{\mathrm{SFG}}^{(\mathrm{coh})},
$$

which shows that the coherent component of the SFG field contains the same kind of information on the biphoton amplitude both in the space-time domain and in the spectral domain. The most important difference lies in the fact that the coherent intensity in the spectral domain is concentrated in a single peak at the origin $(\vec{q}=0, \Omega=0)$, i.e., in the mode corresponding to the original pump field. On the other hand, the spectrum of the incoherent background is $\delta$ correlated in space and time, and spreads over a very broad range of spatial and temporal frequencies, its particular shape being related to the phase-matching conditions inside the SFG crystal as elucidated in Ref. [20]. This circumstance suggests that either far-field or spectral measurement may be conveniently used to enhance the visibility of the coherent contribution with respect to the incoherent background.

\section{RETRIEVING THE PDC CORRELATION}

Let us now investigate how the information on the correlation $\psi_{\mathrm{PDC}}(\vec{x}, t)$ of twin beams and twin photons can be effectively extracted from the coherent component of the SFG light, given by Eq. (43b). Making explicit the dependence on the temporal delay and spatial shift applied to beam + and beam - contained in the transfer functions, (27), it can be written in the form

$$
I_{\mathrm{SFG}}^{(\mathrm{coh})}[\Delta \vec{s}, \Delta t]=\left|\psi_{\text {meas }}(\Delta \vec{s}, \Delta t)\right|^{2},
$$

where

$$
\psi_{\text {meas }}(\Delta \vec{\xi})=2 \int \frac{d \vec{w}}{(2 \pi)^{3}} \Theta\left(q_{y}\right) e^{i \vec{w} \cdot \Delta \vec{\xi}} F_{\mathrm{PDC}}(\vec{w}) F_{\mathrm{SFG}}^{(\mathrm{coh})}(\vec{w})
$$

and $\Delta \vec{\xi} \equiv(\Delta \vec{s}, \Delta t)$.

In this section we consider the configuration shown in Fig. 4(a), where mirror M1 is rotated orthogonally to the gap between the two movable mirrors so that $\Delta \vec{s} \equiv(\Delta x=$ $\left.2 f \Delta \phi_{x}, 0\right)$. In this case, the right-hand side of Eq. (45) coincides with the square modulus of the following quantity:

$$
\begin{aligned}
& \psi_{\text {meas }}(\Delta x, 0, \Delta t) \\
& =\int \frac{d \vec{q}}{(2 \pi)^{2}} \int \frac{d \Omega}{2 \pi} e^{i q_{x} \Delta x-i \Omega \Delta t} F_{\mathrm{PDC}}(\vec{q}, \Omega) F_{\mathrm{SFG}}^{(\mathrm{coh})}(\vec{q}, \Omega) .
\end{aligned}
$$



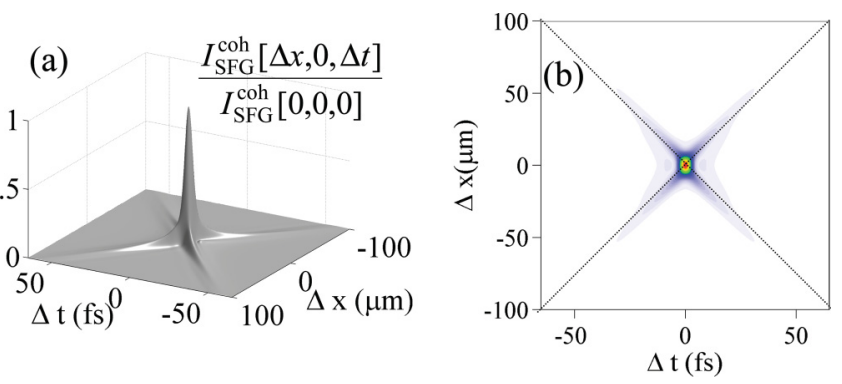

FIG. 5. (Color online) (a) Surface and (b) density plots $I_{\mathrm{SFG}}^{\mathrm{coh}}[\Delta x, 0, \Delta t]=\left|\psi_{\text {meas }}(\Delta x, 0, \Delta t)\right|^{2}$ normalized to the peak value, corresponding to measurement in the configuration in Fig. 4(a).

The step function $\Theta\left(q_{y}\right)$ could be eliminated from Eq. (46) by exploiting the parity of the integrand with respect to $q_{x}$, the latter being a consequence of the radial symmetry of $F_{\mathrm{PDC}}(\vec{q}, \Omega)$ and $F_{\mathrm{SFG}}^{(\mathrm{coh})}(\vec{q}, \Omega)$ in the $\left(q_{x}, q_{y}\right)$ plane. The function $\psi_{\text {meas }}(\Delta x, 0, \Delta t)$, like $\psi_{\mathrm{PDC}}$, is therefore an even function of both the spatial and the temporal coordinates, a feature not holding for the alternative configuration with mirror M1 rotating around the $x$ axis [see Fig. 4(b)], which is discussed in the next section. Figure 5 plots the coherent contribution of the SFG intensity as a function of the applied spatial and the temporal shifts. In this example, the two crystals have the same lengths, $l_{c}=l_{c}^{\prime}=4 \mathrm{~mm}$, and the PDC gain is $g=8$. The structure preserves the nonfactorable $\mathrm{X}$-shaped geometry of the original PDC correlation function $\left|\psi_{\mathrm{PDC}}\right|^{2}$ shown in Fig. 3, with symmetric tails developing along the bisector lines $\Delta t= \pm \sqrt{k_{1}^{\prime \prime} k_{1}} \Delta x$ (corresponding to the diagonals of the plot frame).

The SFG coherent component, (47), differs from the PDC biphoton correlation $\left|\psi_{\mathrm{PDC}}\right|^{2}$ defined in Eq. (16) only for the presence of the SFG spectral amplitude $F_{\mathrm{SFG}}^{(\mathrm{coh})}(\vec{w})$. The latter describes how the phase-matching mechanism selects the spatiotemporal modes in the coherent up-conversion process. We analyze in detail how it affects the PDC correlation measurement in the next subsection. In this regard, it is useful to recast Eq. (46) in the form of a convolution of the PDC biphoton amplitude $\psi_{\mathrm{PDC}}$, the quantity under investigation, with the Fourier transform of $\Theta\left(q_{y}\right) F_{\mathrm{SFG}}^{(\mathrm{coh})}(\vec{q}, \Omega)$, i.e.,

$$
\psi_{\text {meas }}(\Delta \vec{\xi})=\int d \vec{\xi}^{\prime} \psi_{\mathrm{SFG}}\left(\Delta \vec{\xi}-\vec{\xi}^{\prime}\right) \psi_{\mathrm{PDC}}\left(\vec{\xi}^{\prime}\right),
$$

where

$$
\psi_{\mathrm{SFG}}(\Delta \vec{\xi})=\int \frac{d \vec{\xi}}{(2 \pi)^{3}} e^{i \vec{w} \cdot \Delta \vec{\xi}} F_{\mathrm{SFG}}^{(\mathrm{coh})}(\vec{w}) .
$$

This identity has been written taking into account that $\Delta \vec{s} \equiv$ $(\Delta x, 0)$, so that the step function could be eliminated as for Eq. (47). Noting that Eqs. (49) and (38) are formally identical to Eqs. (16) and (14), we see that $\psi_{\mathrm{SFG}}(\Delta \vec{\xi})$ is proportional to the low-gain biphoton amplitude in the space-time domain for the second SFG crystals. It represents the probability amplitude that a pair of photons delayed by a time $\Delta t$ and displaced by $\Delta \vec{s}$ is coherently back-converted into the plane-wave pump mode $(\vec{q}=0, \Omega=0)$. This function can also be interpreted as the optical response function of the SFG crystal in the measurement of $\left|\psi_{\mathrm{PDC}}\right|^{2}$ via SFG.

\section{A. Thin SFG crystal limit}

Let us assume that the two crystals are tuned for the same phase-matching conditions, so that $\Delta^{\mathrm{sfg}}(\vec{w})=\Delta^{\mathrm{pdc}}(\vec{w})$, but the SFG crystal is much shorter than the PDC crystal, $l_{c}^{\prime} \ll$ $l_{c}$. In this limit, the PDC biphoton amplitude can be exactly reconstructed by monitoring the SFG coherent component.

This can be shown by inspection of Eq. (46). When the same phase-matching conditions hold in the two crystals, the spectral probability amplitudes $F_{\mathrm{SFG}}^{(\mathrm{coh})}(\vec{w})$ and $F_{\mathrm{PDC}}(\vec{w})$ are peaked around the same geometrical curve $\Delta^{\mathrm{pdc}}(\vec{w})=$ $\Delta^{\mathrm{sfg}}(\vec{w})=0$. However, for $l_{c}^{\prime} \ll l_{c}$ the spectral bandwidths in the SFG crystal are much wider than in the PDC crystal [the bandwidths of $F_{\mathrm{SFG}}$ exceeds those of $F_{\mathrm{PDC}}$ by a factor of $\sqrt{l_{c} / l_{c}^{\prime}} \gg 1$, according to Eq. (19)]. As a result, $F_{\mathrm{SFG}}^{(\mathrm{coh})}(\vec{w})$ is almost constant in the region where $F_{\mathrm{PDC}}(\vec{w})$ is not negligible, close to its maximum value $\sigma l_{c}^{\prime}$. Under these conditions, the filtering effect due to phase matching in the SFG crystal becomes ineffective and we have

$$
I_{\mathrm{SFG}}^{(\mathrm{coh})}[\Delta x, 0, \Delta z] \approx \sigma^{2} l_{c}^{\prime 2}\left|\psi_{\mathrm{PDC}}(\Delta x, 0, \Delta t)\right|^{2} \quad\left(l_{c}^{\prime} \ll l_{c}\right) .
$$

Thus the coherent component of the SFG output reproduces the biphoton correlation as anticipated.

The same conclusion can be derived also looking at Eq. (48): in the limit $l_{c}^{\prime} \ll l_{c}$, the biphoton amplitude $\psi_{\mathrm{SFG}}$, defined by Eq. (49), has scales of variation in space and time much shorter than $\psi_{\mathrm{PDC}}$, because it is the Fourier transform of a much wider spectral amplitude. It therefore behaves as a $\delta$ function inside the convolution integral, (48), so that the PDC biphoton amplitude is recovered.

For completeness, we mention that in the $l_{c}^{\prime} \rightarrow 0$ limit the incoherent contribution, (43a), takes the form

$$
I_{\mathrm{SFG}}^{\text {(inc) }}=\sigma^{2} l_{c}^{\prime 2}\left|\left\langle b_{1}^{\dagger}(\vec{x}, t) b_{1}(\vec{x}, t)\right\rangle\right|^{2} \quad\left(l_{c}^{\prime} \rightarrow 0\right),
$$

where

$$
\left\langle b_{1}^{\dagger}(\vec{x}, t) b_{1}(\vec{x}, t)\right\rangle=\int \frac{d \vec{q}}{(2 \pi)^{2}} \int \frac{d \Omega}{(2 \pi)}|V(\vec{q}, \Omega)|^{2}
$$

is the PDC photon flux evaluated at the output face of the first crystal [as can be easily inferred from relation (9a)]. However, we see in Sec. VII that the temporal walk-off between the PDC field and the generated incoherent SFG field plays a fundamental role in the formation of the incoherent component even when considering short propagation distances. In practice, the validity of expressions (51) fails as soon as the finite length of the SFG crystal is taken into account.

\section{B. Long SFG crystal}

We now investigate how propagation in the SFG crystal affects the retrieval of the PDC correlation via the measurement of $I_{\mathrm{SFG}}^{\text {(coh) }}[\Delta x, 0, \Delta z]$, showing that both the X-shaped geometry and the strong localization of the biphoton correlation are preserved, at least when the two crystals are equally tuned.

The left panels in Fig. 6 plot the retrieved quantity $I_{\mathrm{SFG}}^{(\mathrm{coh})}[\Delta x, 0, \Delta t]$ for increasing values of the SFG crystal length $l_{c}^{\prime}$. The most evident effect is that the tails of the correlation becomes progressively more visible with respect to the central peak, as the propagation distance inside the SFG crystal 
$\frac{I_{S F G}^{c o h}[\Delta x, 0, \Delta t=\Delta z / c]}{I_{S F G}^{c o h}[0,0,0]}$

temporal cross-section for $\Delta x=25 \mu \mathrm{m}$

(a) $l_{\mathrm{c}}^{\prime}=100 \mu \mathrm{m}$
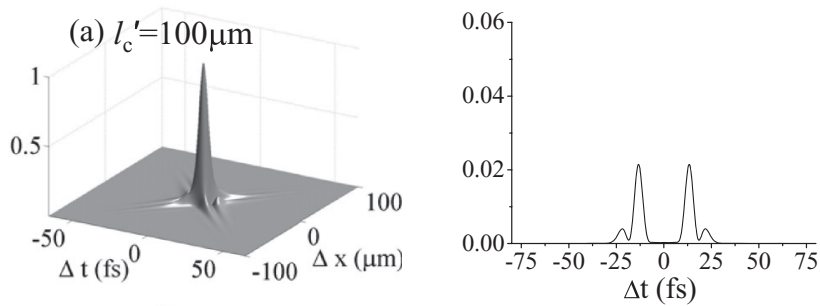

(b) $l_{\mathrm{c}}^{\prime}=2000 \mu \mathrm{m}$

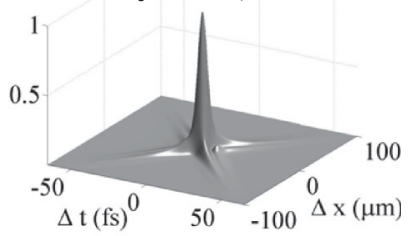

(c) $l_{\mathrm{c}}{ }^{\prime}=4000 \mu \mathrm{m}$
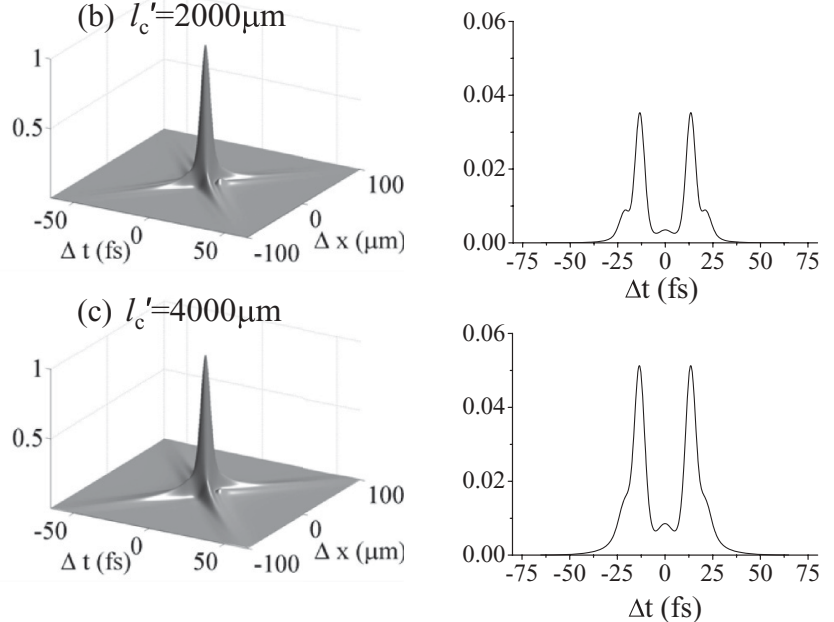

FIG. 6. Left: Numerical evaluation from Eqs. (45)-(47) of $I_{\mathrm{SFG}}^{\mathrm{coh}}[\Delta x, 0, \Delta t] / I_{\mathrm{SFG}}^{\mathrm{coh}}[0,0,0]$ for increasing values of the SFG crystal length $l_{c}^{\prime}$. Right: Corresponding temporal cross section $I_{\mathrm{SFG}}^{\mathrm{coh}}[\Delta x=$ $25 \mu \mathrm{m}, 0, \Delta t] / I_{\mathrm{SFG}}^{\mathrm{coh}}[0,0,0]$. The $\mathrm{X}$-shaped structure is robust against propagation in the SFG crystal and the tails are enhanced with respect to the central peak as $l_{c}^{\prime}$ increases. The PDC parameters are $g=8$, $l_{c}=4 \mathrm{~mm}$.

increases. This is clearly shown by the temporal cross sections at $\Delta x=25 \mu \mathrm{m}$ of the same quantity (right panels in Fig. 6). Figure 7 shows that the height of the tails, at a fixed time delay $\Delta t=30 \mathrm{fs}$, increases almost linearly with $l_{c}^{\prime}$ up to the value of the PDC crystal length $l_{c}=4 \mathrm{~mm}$. We expect therefore that the choice of a few-millimeter-long SFG crystal, with respect to that of a very short crystal, presents a twofold advantage: (i) the total number of up-converted photons is

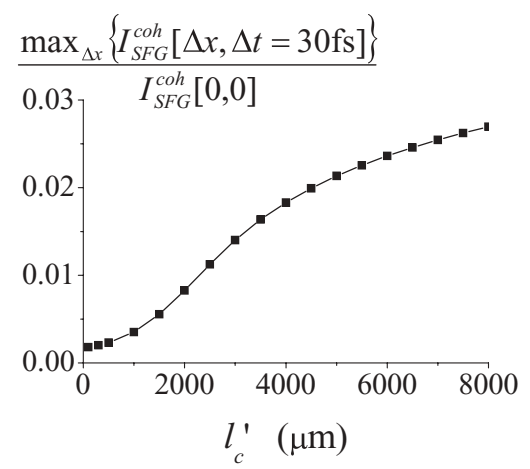

FIG. 7. Plot of the height of the tails of the retrieved correlation $\max _{\Delta x}\left\{I_{\mathrm{SFG}}^{\mathrm{coh}}[\Delta x, 0, \Delta t=30 \mathrm{fs}]\right\}$, at a fixed time delay $\Delta t=30 \mathrm{fs}$, normalized to the peak value $I_{\mathrm{SFG}}^{\mathrm{coh}}[0,0,0]$, as a function of $l_{c}^{\prime}$.

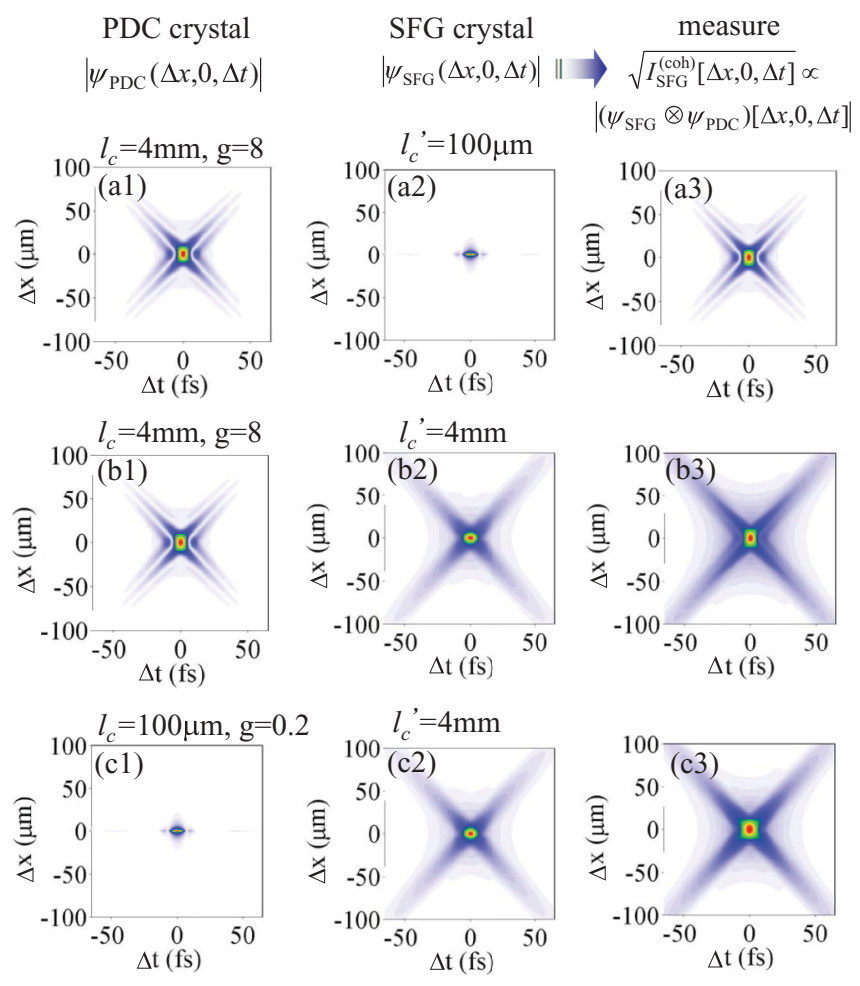

FIG. 8. (Color online) Left and middle panels: Density plots of $\left|\psi^{\mathrm{PDC}}(\Delta x, 0, \Delta t)\right|$ and $\left|\psi^{\mathrm{SFG}}(\Delta x, 0, \Delta t)\right|$, respectively, considering either short or long crystals (values of $l_{c}$ and $l_{c}^{\prime}$ are indicated). Right panel: Square root of the measured quantity, $\sqrt{I_{S F G}^{\text {coh }}}=$ $\left|\psi_{\text {meas }}(\Delta x, 0, \Delta t)\right|$, obtained by convolving $\psi_{\mathrm{PDC}}$ and $\psi_{\mathrm{SFG}}$. See text for discussion.

obviously larger, and (ii) the visibility of the tails, although small with respect to that of the central peak, will be enhanced, a feature which should facilitate the experimental observation of the X-shaped structure through the scanning of $\Delta x$ and $\Delta t$. A further advantage lies in that the overall visibility of the coherent component with respect to the incoherent background improves substantially as the SFG crystal length is increased above a few hundred micrometers, as shown in Sec. VII.

An explanation of the behavior depicted in Fig. 6 can be obtained considering the convolution integral in Eq. (48) and referring to the density plots in Fig. 8, which illustrate the effect of the convolution (48) under three different conditions.

(a) $l_{c}^{\prime} \ll l_{c}$ : The SFG crystal response function $\psi_{\mathrm{SFG}}$ behaves as a Dirac $\delta$ function both in the spatial and in the temporal domain [see Fig. 8 (a2)], so that the second crystal works as an ultrafast correlator with an instantaneous and localized response. The coherent component of the SFG intensity [Fig. 8(a3)] therefore provides the PDC biphoton amplitude square modulus $\left|\psi_{\mathrm{PDC}}\right|^{2}$, in agreement with the short crystal limit result, (50).

(b) $l_{c} \sim l_{c}^{\prime}$ : If the SFG crystal has a length comparable to that of the PDC crystal, it displays a nonlocal spatiotemporal response. However, provided the two crystals are tuned for the same phase-matching conditions, the response of the SFG crystal has the same geometrical $\mathrm{X}$ shape as the original biphoton correlation [see Figs. 8(b1) and 8(b2)]. This is not unexpected: as a matter of fact, the function $\psi_{\mathrm{SFG}}$ is identical to 

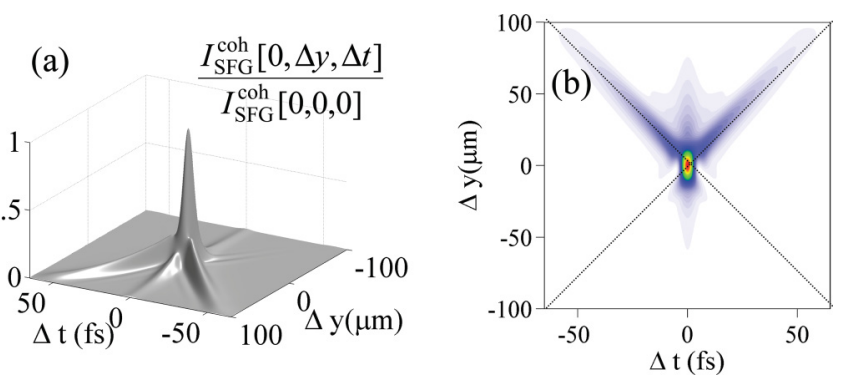

FIG. 9. (Color online) (a) Surface and (b) density plots $I_{\mathrm{SFG}}^{\mathrm{coh}}[0, \Delta y, \Delta t]=\left|\psi_{\text {meas }}(0, \Delta y, \Delta t)\right|^{2}$ normalized to the peak value, corresponding to a measurement in the configuration in Fig. 4(a).

$\psi_{\text {PDC }}$ in the low-PDC-gain limit (apart from a constant factor). In the high-gain regime of PDC considered in this example, the PDC biphoton amplitude displays a faster decay of the tails than its low-gain counterpart ${ }^{4}$ but, nevertheless, keeps the same geometrical shape. Therefore $\psi_{\text {meas }}$ is the convolution of two functions which have almost the same $\mathrm{X}$-shaped structure in the space-time domain, and the main effect of this convolution is to enhance the weight of the tails with respect to the central peak, as can be seen from Fig. 8(b3). Most importantly from our point of view, the nonfactorable $\mathrm{X}$-shaped geometry of the PDC biphoton amplitude is preserved, as well as its strong localization in time (or in space) when particular temporal (or spatial) cross sections are considered.

(c) $l_{c} \ll l_{c}^{\prime}$ : Because of the symmetrical role played by $\psi_{\mathrm{PDC}}$ and $\psi_{\mathrm{SFG}}$ in Eq. (48), a configuration with $l_{c} \ll l_{c}^{\prime}$ would provide a direct measure of $\left|\psi_{\mathrm{SFG}}\right|^{2}$, which is proportional to the PWP biphoton amplitude in the SFG crystal at low parametric gains [accordingly, the density plots, Figs. 8(c2) and 8(c3), are nearly identical, as for plots Figs. 8(a1) and 9(a3) in case a]. However, in this case the SFG photon flux would be strongly reduced because of the lower gain of the PDC field associated with short crystals.

\section{ALTERNATIVE DETECTION SCHEME}

We now focus on the alternative configuration (b) for mirror M1 shown in Fig. 4(b), considering rotations performed around the $x$ axis. In contrast to configuration (a), treated in the previous section, the step function $\Theta\left(q_{y}\right)$ cannot be eliminated from Eq. (46) and the SFG coherent component is now given by the square modulus of

$$
\begin{aligned}
\psi_{\text {meas }}(0, \Delta y, \Delta t)= & 2 \int \frac{d \vec{q}}{(2 \pi)^{2}} \int \frac{d \Omega}{2 \pi} e^{i q_{y} \Delta y-i \Omega \Delta t} \Theta\left(q_{y}\right) \\
& \times F_{\mathrm{PDC}}(\vec{q}, \Omega) F_{\mathrm{SFG}}^{(\mathrm{coh})}(\vec{q}, \Omega),
\end{aligned}
$$

with $\Delta y=2 f \Delta \phi_{y}$.

Because of the presence of the step function, the even symmetry with respect to the spatial coordinate is lost. As shown in Fig. 9, the retrieved correlation function in the $(\Delta t, \Delta y)$ plane displays a V-shaped geometry: the tails extend

\footnotetext{
${ }^{4}$ The faster decay of the PDC correlation $\psi_{\mathrm{PDC}}$ along its tails for high gain is due to the broadening of the spectral amplitude with $g$ described by Eq. (20).
}

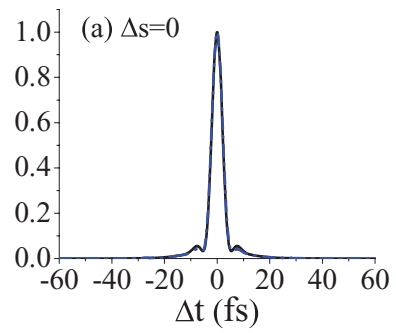

$$
\begin{gathered}
-\frac{I_{\mathrm{SFG}}^{\mathrm{coh}}[\Delta x=\Delta s, 0, \Delta t]}{I_{\mathrm{SFG}}^{\mathrm{coh}}[0,0,0]} \\
\cdots \cdot \frac{I_{\mathrm{SFG}}^{\mathrm{coh}}[0, \Delta y=\Delta s, \Delta t]}{I_{\mathrm{SFG}}^{\mathrm{coh}}[0,0,0]}
\end{gathered}
$$
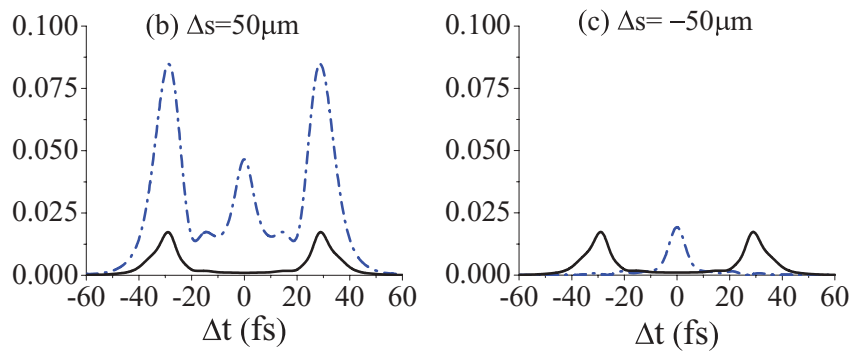

FIG. 10. (Color online) Temporal cross sections of the SFG coherent component obtained by varying $\Delta t$ for (a) $\Delta s=0$, (b) $\Delta s=50 \mu \mathrm{m}$, and (c) $\Delta s=-50 \mu \mathrm{m}$. The solid line corresponds to configuration (a); the dotted-dashed line corresponds to configuration (b).

along the lines $\Delta t= \pm \sqrt{k_{1}^{\prime \prime} k_{1}} \Delta y$ in the $\Delta y>0$ half-plane, while they disappear for $\Delta y<0$. Moreover, compared to the result obtained in configuration (a) shown in Fig. 5, the tails are strongly enhanced with respect to the central peak. From simple geometrical optics considerations, it can be shown that negative values of the rotation angle $\Delta \phi_{y}$ of mirror M1 around the $x$ axis prevents the overlap of beam + and beam-inside the SFG crystal, so that the up-conversion efficiency becomes much lower under this condition (i.e., for $\Delta y<0$ ). On the contrary, a negative rotation $\Delta \phi_{y}$ increases the overlap of beam + and beam -thereby enhancing the up-conversion efficiency compared to configuration (a) in the $\Delta y>0$ region.

Noting that the complex amplitude $\psi_{\text {meas }}(\Delta x, 0, \Delta t)$ and $\psi_{\text {meas }}(0, \Delta y, \Delta t)$ given by Eqs. (47) and (53) are linked through the relation

$$
\begin{aligned}
\psi_{\text {meas }}(\Delta x, 0, \Delta t)= & \frac{1}{2}\left[\psi_{\text {meas }}(0, \Delta y=\Delta x, \Delta t)\right. \\
& \left.\times \psi_{\text {meas }}(0, \Delta y=-\Delta x, \Delta t)\right],
\end{aligned}
$$

from which it can be inferred that the temporal profile across the central peak [obtained by setting $\Delta x=0$ in case (a) and $\Delta y=0$ in case (b)] is identical in the two configurations [see Fig. 10(a)]. On the other hand, $\psi_{\text {meas }}(0, \Delta y, \Delta t) \approx 0$ in the $\Delta y<0$ region being not too close to the central peak, relation (54) implies that $I_{\mathrm{SFG}}^{\mathrm{coh}}[0, \Delta y, \Delta t] \approx 0$ for $\Delta y<0$ and $I_{\mathrm{SFG}}^{\mathrm{coh}}[0, \Delta y, \Delta t] \approx 4 \times I_{\mathrm{SFG}}^{\mathrm{coh}}[\Delta x=\Delta y, 0, \Delta t]$ for $\Delta y>0$. The tails of the coherent SFG component, extending only in the $\Delta y>0$ region in configuration (b), are thus about four times more intense than in configuration (a). This behavior is shown in Figs. 10(b) and 10(c), which compare the temporal profile in the two configurations for the spatial shifts $\Delta s=50 \mu \mathrm{m}$ and $\Delta s=-50 \mu \mathrm{m}$, respectively.

We note that configuration (b) offers the relevant benefit that the tails are more visible and can therefore be detected more easily than in configuration (a). Although, in principle, configuration (b) does not allow the reconstruction of the 
symmetric $\mathrm{X}$-shaped correlation as configuration (a), we found the following approximate empirical relation that links the results in the two configurations:

$$
\begin{aligned}
I_{\mathrm{SFG}}^{\mathrm{coh}}[\Delta x= & \Delta y, 0, \Delta t] \\
\approx & \left.\frac{1}{4}\left[\sqrt{I_{\mathrm{SFG}}^{\mathrm{coh}}[0, \Delta y, \Delta t]}-\sqrt{I_{\mathrm{SFG}}^{\mathrm{coh}}[0,-\Delta y, \Delta t}\right]\right]^{2} \\
& \text { for } \quad \Delta y \neq 0,
\end{aligned}
$$

this approximation holding as long as $\Delta y$ is sufficiently large, i.e., far from the central peak. This relation should allow us to infer from experimental data collected in configuration (b) the data that would be collected in configuration (a), thus allowing the reconstruction of the X-shaped PDC correlation in configuration (b).

\section{VISIBILITY OF THE INFORMATION}

The issue of the visibility of the information contained in the coherent SFG contribution, against the incoherent background, is a crucial one, especially for the observation of the tails of the PDC X-shaped correlation. As already remarked, expression (37) of the spectral coherence function of the SFG light suggests that the visibility should be greatly enhanced by measuring the SFG light in the far field of the second crystal, where the coherent contribution propagating in the forward direction is separated from the incoherent background propagating over a broad angle. This issue is discussed in Sec. VIIB. We start here by considering the visibility of a measurement of the total number of SFG photons, which provides a useful estimation of the overall weight of the coherent component with respect to the incoherent one.

\section{A. Bucket detection of the SFG photons}

The photon fluxes $I_{\mathrm{SFG}}^{(\mathrm{inc})}$ and $I_{\mathrm{SFG}}^{(\mathrm{coh})}[\Delta x, 0, \Delta t]$ given in Sec. IV, Eqs. (43), have been evaluated at the SFG crystal output face and are uniform in the transverse plane because of the artifact of the plane-wave pump approximation. Within this limit, the ratio

$$
\mathcal{V}=\frac{I_{\mathrm{SFG}}^{(\mathrm{coh})}[0,0, \Delta z=0]}{I_{\mathrm{SFG}}^{\text {(inc) }}+I_{\mathrm{SFG}}^{(\mathrm{cooh})}[0,0, \Delta z=0]}
$$

therefore defines the visibility of the correlation peak at $\Delta x=$ $\Delta t=0$ against the incoherent background either in the case of a near-field detection of the SFG intensity or assuming that all the SFG photons are collected without discrimination (e.g., by using a bucket detector).

Figure 11 plots the visibility $\mathcal{V}$ as a function of the length of the SFG crystal $l_{c}^{\prime}$ for increasing PDC parametric gain, starting from values of $g$ below unity Fig. 11(a) up to very high values [Fig. 11(b)], the latter corresponding to the regime of the experiment reported in Ref. [10]. The contributions of both the coherent and the incoherent components, (43b) and (43a), have been estimated numerically with a Monte Carlo integration, assuming that a 550-nm FWHM temporal bandwidth is selected with a super-Gaussian frequency filter, from 850 up to $1400 \mathrm{~nm}$.

In a regime of low parametric gain, i.e., for $g \ll 1$, the PDC spectral probability amplitude $F_{\mathrm{PDC}}(\vec{w})$ scales as $g$ [see Eq. (14)], while the spectrum $|V(\vec{w})|^{2}$ given by Eq. (13)
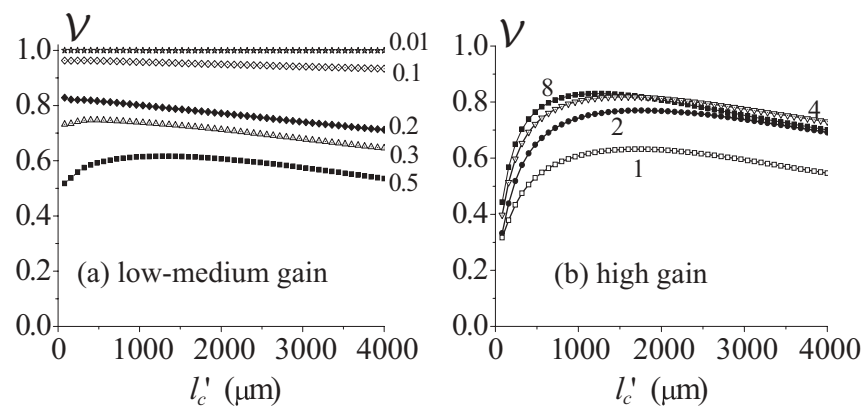

FIG. 11. Visibility of the coherent component with respect to the incoherent background as a function of the length of the SFG crystal $l_{c}^{\prime}$, for (a) medium-low and (b) high parametric gain values (indicated in the figure). The length of the PDC crystal, $l_{c}=4 \mathrm{~mm}$, is kept fixed. The selected temporal bandwidth extends from 850 up to $1400 \mathrm{~mm}$.

scales as $g^{2}$. For this reason, $I_{\mathrm{SFG}}^{(\mathrm{coh})}$ and $I_{\mathrm{SFG}}^{(\mathrm{inc})}$ scale as $g^{2}$ and $g^{4}$, respectively, and the visibility, (56), is close to $100 \%$. This behavior can be physically understood by noting that the probability of finding pair of photons that are not twins becomes exceedingly low in this regime.

As the PDC parametric gain increases, the incoherent component rapidly increases and becomes comparable to the coherent one. As a consequence, $\mathcal{V}$ decreases to lower values, the degradation being particularly relevant in the $l_{c}^{\prime} \ll l_{c}$ limit. For longer propagation distances in the SFG crystal, the generation of incoherent SFG photons becomes less efficient because of spatial walk-off and GVM, while the coherent component is not affected by those phenomena, as further discussed in Ref. [20]. The visibility therefore remains above $60 \%$ even for high PDC gains, as long as the propagation distance inside the SFG crystal exceeds a few hundred micrometers. However, we note that such visibility, although not negligible, would make the detection of the full $\mathrm{X}$ correlation very challenging in a practical implementation, because the tails would be hidden by the incoherent background.

Figure 12 displays $I_{\mathrm{SFG}}^{(\mathrm{coh})}[0,0,0]$ and $I_{\mathrm{SFG}}^{(\mathrm{inc})}$ separately as a function of $l_{c}^{\prime}$, evaluated in the high-gain case $(g=8)$ from Eq. (43). It shows that the coherent component increases almost quadratically up to $l_{c}^{\prime} \sim 1 \mathrm{~mm}$, while for a larger propagation distance in the SFG crystal the coherent up-conversion becomes less efficient. The initial quadratic behavior reflects the result of Eq. (50) derived in the "thin SFG crystal" limit,

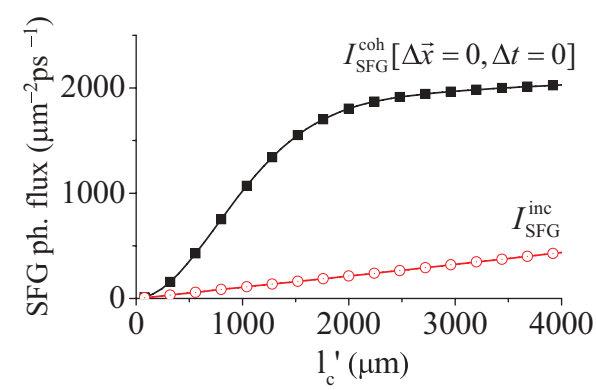

FIG. 12. (Color online) Estimation of the coherent and incoherent SFG photon fluxes, $I_{\mathrm{SFG}}^{(\mathrm{inc})}$ and $I_{\mathrm{SFG}}^{(\mathrm{coh})}[0,0,0]$, as a function of the SFG crystal length $l_{c}^{\prime}$. The PDC crystal parameters are $g=8$ and $l_{c}=$ $4000 \mu \mathrm{m}$. 
where the filtering effect due to the SFG spectral probability amplitude $F_{\mathrm{SFG}}^{\text {(coh) }}(\vec{q}, \Omega)$ in Eq. ( $\left.43 \mathrm{~b}\right)$ is almost ineffective.

By inspecting the expression of the typical bandwidths of $F_{\mathrm{SFG}}^{\text {(coh) }}(\vec{q}, \Omega)$ and $F_{\mathrm{PDC}}(\vec{q}, \Omega)$ [only the latter depends on the parametric gain according to Eq. (20)], we find that they become comparable for $l_{c}^{\prime}=l_{c} \sqrt{\pi^{2} /\left(\pi^{2}+g^{2}\right)} \approx 1.5 \mathrm{~mm}$ for $g=8, l_{c}=4 \mathrm{~mm}$. For $l_{c}^{\prime}$ above this characteristic length the SFG spectral probability amplitude $F_{\mathrm{SFG}}^{(\mathrm{coh})}(\vec{q}, \Omega)$ becomes narrower than $F_{\mathrm{PDC}}(\vec{q}, \Omega)$, and the integral, $(43 \mathrm{~b})$, spans a volume in the Fourier space $\propto l_{c}^{\prime-3 / 2}$, so that $I_{\mathrm{SFG}}^{(\mathrm{coh})}$ not longer scales as $l_{c}^{\prime 2}$.

On the other hand, the SFG incoherent component increases only linearly with $l_{c}^{\prime}$, except for very short propagation distances, for which approximation (51) holds. This is due to the fact that the up-conversion probability $\left|F_{\mathrm{SFG}}\left(\vec{w}, \vec{w}^{\prime}\right)\right|^{2}$ appearing inside the integral on the right-hand side of (43a) decays rapidly when $\vec{w}^{\prime} \neq-\vec{w}$, because of the effect of the spatial walk-off and the temporal group velocity mismatch arising between the ordinary fundamental beam and the extraordinary up-converted beam. These issues are further elucidated in a related publication [20].

\section{B. Far-field detection of the SFG light}

According to the plane-wave pump result of (37), the spectral distribution of the PDC light consists of a coherent contribution, concentrated in the original plane-wave pump mode at $\vec{q}=0$ and $\Omega=0$, superimposed on an incoherent background that spreads over a large bandwidth of spatiotemporal modes. The PWP approximation leads to artificial divergences (the $\delta$-function factors) that do not allow a direct evaluation of the visibility of the coherent component against the incoherent background in the Fourier domain. For this reason, we implemented a full $3 \mathrm{D}+1$ numerical simulation of our proposed setup (see Fig. 3) and modeled the result of the detection of the SFG intensity distribution in the far field of the SFG crystal, as shown in Fig. 13(a). The generation of the broadband PDC field is simulated in the framework of the Wigner representation as described in Ref. [21], taking into account both the finite cross section $w_{p}$ and the duration $\tau_{p}$ of the pump pulse and the phase-matching conditions inside the two crystals (the full BBO Sellmeier dispersion relation [22] is used in the simulation). The propagation in the two crystals, described by Eqs. (4) and (30), is simulated through a pseudospectral (split-step) method using a $256 \times 256 \times 512$ numerical grid in the $(\vec{x}, t)$ and the $(\vec{q}, t)$ spaces. The parameters of the numerical simulations are chosen to reproduce the conditions of the experiment being developed in Como [10], which operates in a pulsed regime of high parametric gain $(g \approx$ 8 ). The broadband PDC field injected into the SFG crystal after the extraction of the pump beam undergoes the three-wave mixing process described by Eqs. (30) and the up-converted SFG field is mapped into the far field with an $f$ - $f$ lens system, as shown schematically in Fig. 13(a). We expect that a single stochastic realization of our simulations roughly reproduces the field distribution obtained from a single pump shot.

The typical far-field intensity distribution of the upconverted SFG field obtained in the detection plane from a Gaussian pump pulse of waist $w_{p}=600 \mu \mathrm{m}$ and duration $\tau_{p}=1 \mathrm{ps}$ is shown in Figs. 13(b) and 13(c). We verified that for the chosen PDC gain $g=8$ the injected PDC field (not shown in the figure) is only slightly depleted during propagation in the second crystal $(<0.1 \%)$, a feature which confirms the validity of the perturbative approximation, (32b), for the SFG field used in the PWPA model. The narrow central peak results from coherent processes in which pairs of phase conjugate photons back-convert to a coherent field component reproducing partially the far-field distribution of the original pump beam. This peak is fixed, in the sense that it is reproduced identically in each stochastic simulation. The broad speckled background instead is noisy and changes in each realization, giving rise, on average, to a broad distribution. It originates from the incoherent processes where non-phase-conjugated photon pairs are up-converted. These kind of simulations reproduce very closely the results on the far-field detection of SFG reported in Ref. [10] (see, in particular, Fig. 4 in Ref. [10]).

The full-scale plot in Fig. 13(c) shows that under such ideal conditions (perfect imaging, no dispersive optical elements, no misalignments between the two crystals), the visibility of the coherent vs incoherent component for $\Delta x=\Delta t=0$ is very high, close to $100 \%$. We can therefore expect that this far-field detection scheme is well suited for performing the measurement of the full $\mathrm{X}$ correlation.

Figure 14 simulates the reconstruction of the $\mathrm{X}$-shaped correlation obtained by monitoring the number of photons in the central peak of the SFG far field as a function of the temporal delay $\Delta t$ and the spatial shift $\Delta x$ imposed on the two twin PDC components (more precisely, it reports the number of SFG photons over a pixel $56 \times 56 \mu \mathrm{m}$ wide, in the focal plane of a lens with $f=20 \mathrm{~cm}$, placed at a focal distance from the SFG crystal). We verified that for the chosen pump pulse parameters, the retrieved structure in the $(\Delta t, \Delta x)$ plane is very close to that obtained by evaluating the coherent component, (47), within the PWPA [see, e.g., Fig. 9(a)]. Note that despite the fact that both the coherent and the incoherent components are displayed in Fig. 14, the visibility of the tails of the correlation with respect to the speckled background is very high. The relative weight of the incoherent background, about $0.2 \%$ of the coherent peak value, can be inferred from the height of the baseline of the temporal profile plotted in Fig. 14(d) for $\Delta x=0$.

\section{FRAGILITY OF THE CORRELATION MEASUREMENT}

The previous simulations that display nearly $100 \%$ visibility for the correlation measurement have been obtained assuming a perfect imaging of the PDC source into the SFG crystal input face. The coherent component $\propto\left|\psi_{\text {meas }}(\Delta x, 0, \Delta t)\right|^{2}$ is, however, strongly phase sensitive and, therefore, extremely fragile against dispersion, imperfect imaging conditions, and small misalignments between the two crystals. For this reason, the slightest imperfection in the imaging device that maps the PDC output plane into the SFG input plane deteriorates the coherent component of the SFG field which contains the correlation information. On the other hand, the incoherent component, (43a), remains unaffected because of its phase-insensitive nature, so that the overall visibility of 

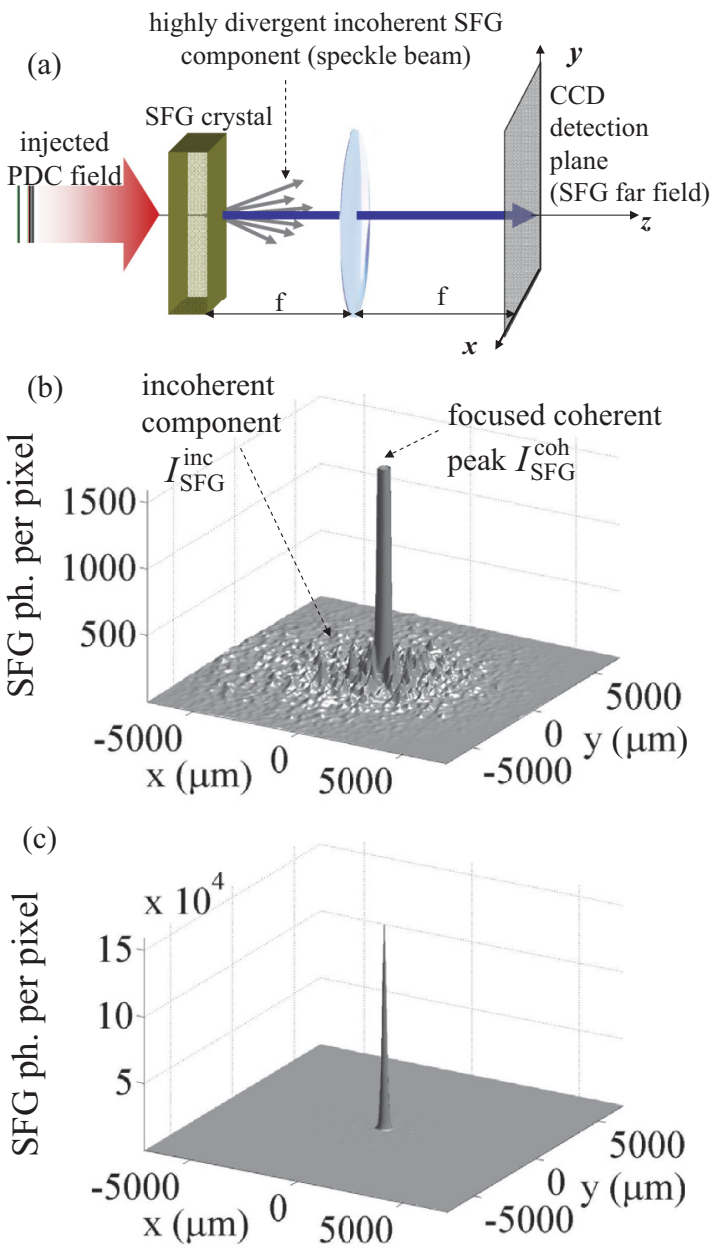

FIG. 13. (Color online) Numerical 3D +1 modeling of the experiment. (a) Detection scheme with a $2 f$ lens system to observe the SFG far field $(f=20 \mathrm{~cm}$ ). (b) Far-field distribution of the SFG light for $\Delta \vec{x}=\Delta t=0$ : the narrow central peak (truncated to $1 \%$ of its peak value) is the coherent component, arising from the up-conversion of phase-conjugated photons. The incoherent contribution gives rise to the broad speckled distribution. (c) Full-scale plot, showing that the visibility of the coherent component is close to $100 \% . g=8$, $w_{p}=600 \mu \mathrm{m}, \tau_{p}=1 \mathrm{ps}$. The $z$ axis scale gives the estimated number of photons on the $56 \times 56 \mu \mathrm{m}$ pixels of the numerical grid.

$\left|\psi_{\text {meas }}(\Delta x, 0, \Delta t)\right|^{2}$ is strongly sensitive to those imperfections. In the following we study the effects of the main sources of experimental imperfection.

\section{A. Temporal dispersion}

It is well known $[14,15,17,18]$ that the scheme is very sensitive to the presence of any dispersive optical elements. As an example, Fig. 15 shows the effect of a half-millimeter-thick slab of BK7 glass inserted in the propagation path between the PDC and the SFG crystals.

It shows that not only is the central correlation peak broadened, but also the X-shaped structure of the correlation is strongly distorted. As a matter of fact, in order to compensate for the detrimental effect of dispersion introduced by optical lenses, prisms have been used in experimental works $[14,15,17]$; a valid alternative approach, which we
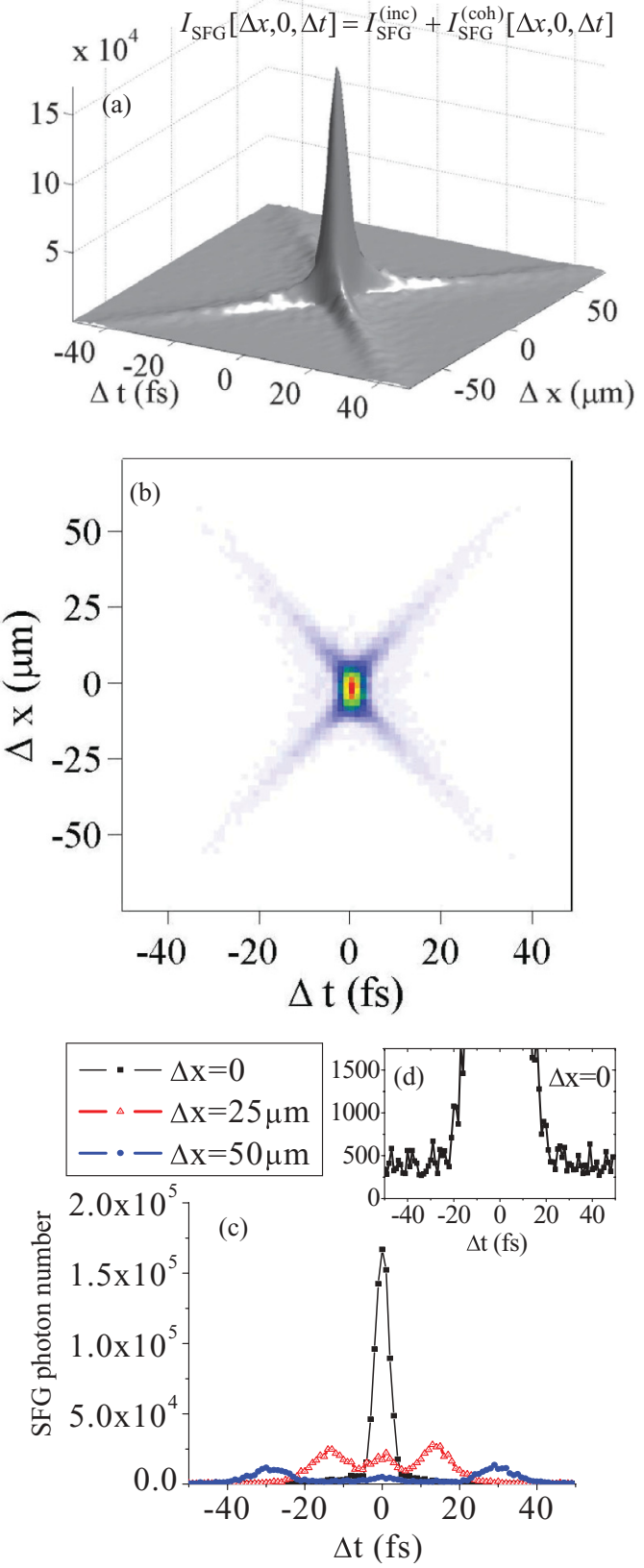

FIG. 14. (Color online) Simulation of the reconstruction of the $\mathrm{X}$ correlation via SFG under ideal conditions based on the far-field detection scheme in Fig. 13(a). (a) Surface and (b) density plots of the number of SFG photons measured in the central pixel (at $\vec{x}=0$ ), obtained by scanning $\Delta x$ and $\Delta t$ on an $80 \times 80$ grid. (b) Temporal profiles for three values of $\Delta x$ showing the transition from the central-peak to the double-peak profile. (d) Baseline of the $\Delta x=0$ profile, corresponding to the incoherent background with around 350 photons per pixel, about $0.2 \%$ of the peak value at $\Delta x=\Delta t=0 . g=8, w_{p}=600 \mu \mathrm{m}, \tau_{p}=1 \mathrm{ps}$.

implemented in Refs. [10] and [11] is, rather, to replace dispersive lenses with achromatic parabolic mirrors.

\section{B. Imperfect imaging}

Because of the smallness of the variation scale of the $\mathrm{X}$ correlation along the spatial dimension $(\lesssim 10 \mu \mathrm{m})$, we expect 

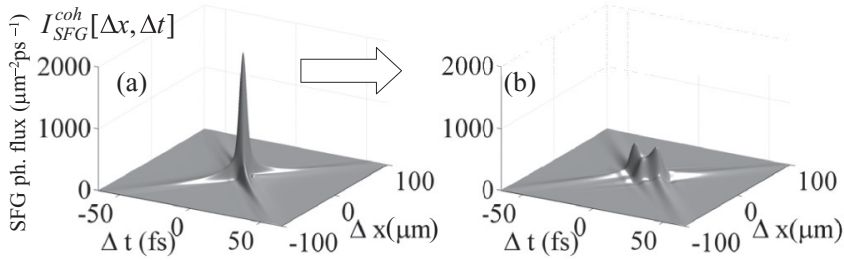

FIG. 15. Simulation of the effect of a dispersive element between the PDC and the SFG crystal obtained using the PWPA model. Spatiotemporal correlation $\left|\psi_{\text {meas }}\right|^{2}$ reconstructed (a) under ideal conditions, (b) in the presence of a $0.5-\mathrm{mm}$ slab of BK7 glass between the PDC and the SFG crystals.

that the scheme shows very little tolerance with respect to errors in the positioning of the SFG crystal with respect to the image plane of the telescopic system illustrated in Fig. 1. Under ideal imaging conditions, the spatial width of the correlation peak is indeed determined by the collected PDC bandwidth considered in the simulation, i.e., $2 q_{\max } \approx 0.6 \mu \mathrm{m}^{-1}$, which gives a spatial correlation profile of width $\Delta x_{\mathrm{fwhm}} \approx 11 \mu \mathrm{m}$ [see Figs. 2(a) and 3(c)].

Let us suppose, for example, that the second crystal entrance face is set at a distance $f+\Delta z_{\text {img }}$ from the second lens of the $4 f$ system. This error introduces an additional phase factor to the transfer function product that enters into Eq. (43b), which is now given by

$$
H_{+}(\vec{q}, \Omega) H_{-}(-\vec{q},-\Omega)=e^{-i \Omega \Delta t} e^{i q_{x} \Delta x} e^{-i \frac{c q^{2}}{\omega_{1}\left(1-\Omega^{2} / \omega_{1}^{2}\right)} \Delta z_{\mathrm{img}}} .
$$

The condition for neglecting the diffraction term reads

$$
\Delta z_{\text {img }} \ll z_{\text {DOF }} \equiv \frac{\pi^{2}}{\lambda q_{\max }^{2}}\left(1-\frac{\Omega_{\max }^{2}}{\omega_{1}^{2}}\right),
$$

where $q_{\text {max }}$ is the maximal transverse wave vector of the PDC emission in the collected bandwidth. For the temporal bandwidth $2 \Omega_{\max }=0.965 \times 10^{15} \mathrm{~Hz}$ assumed in the simulations, we have a tolerance on $\Delta z_{\text {img }}$ of the order of hundreds of micrometers.

This is confirmed by our numerical modeling of the experiment. The first effect of an error in the imaging system is that the efficiency of the coherent up-conversion drops substantially with respect to the ideal configuration. Figure 16 shows the far-field distribution of the SFG light [as it would be detected in the scheme in Fig. 13(a)], for increasing values of the error in the imaging plane $\Delta z_{\text {img. }}$. A displacement of $500 \mu \mathrm{m}$ is sufficient to decrease the back-conversion efficiency of the twin photons by a factor of 12 , and for $\Delta z_{\mathrm{mm}}=2 \mathrm{~mm}$ the coherent peak becomes comparable in magnitude to the incoherent background (which is not sensitive to $\Delta z_{\text {img }}$ ).

Even more dramatic is the deterioration of the shape of the correlation function. Figure 17 plots the spatiotemporal profile of the detected PDC correlation $\left|\psi_{\text {meas }}(\Delta x, 0, \Delta t)\right|^{2}$ [rows (a) and (b)] and of its temporal cross section at $\Delta x=0$ [row (c)] for increasing values of the error $\Delta z_{\text {img }}$, evaluated from the PWPA result, (43b). The density plots in row (a) show that a large broadening occurs along the spatial direction. This is not unexpected: with respect to an error in the imaging plane, the reconstructed spatial correlation as a function of $\Delta x$ behaves
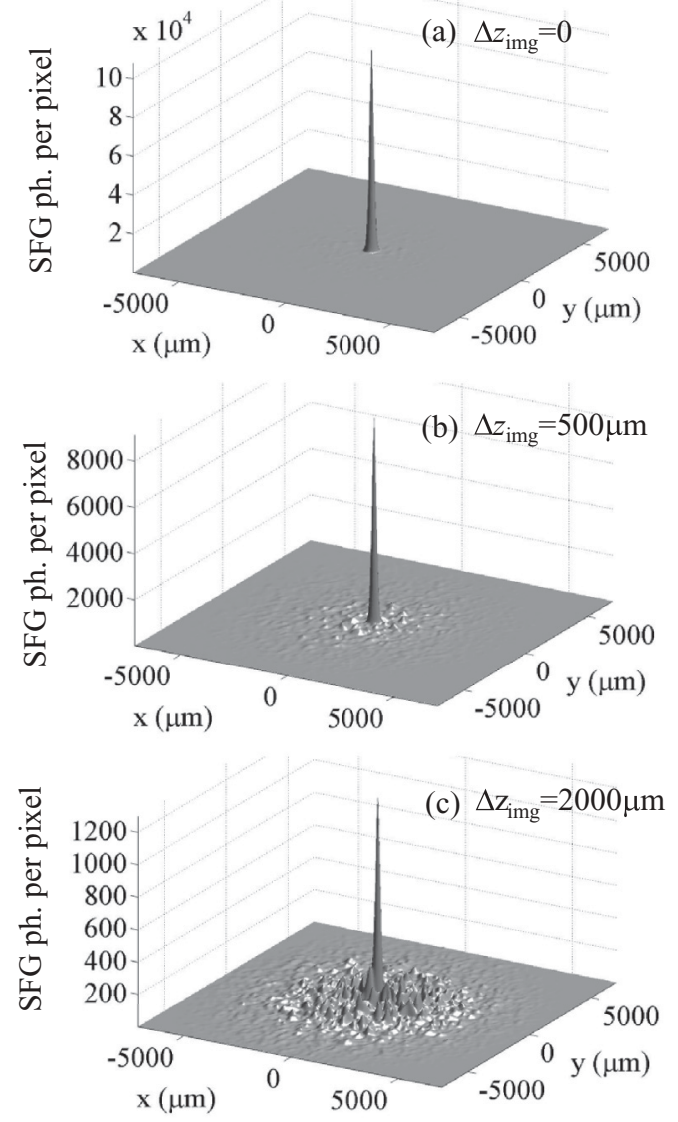

FIG. 16. Simulation of the far-field distribution of the SFG light evaluated for increasing values of the error in the imaging plane positioning $\Delta z_{\text {img }}$.

as the spatial resolution of an ordinary optical image with respect to the depth of focus of the imaging system, i.e., it roughly broadens as $\Delta x_{\mathrm{fwhm}} \sqrt{1+\Delta z_{\mathrm{img}}^{2} / z_{\mathrm{DOF}}^{2}}$.

Less expected is perhaps the broadening of the correlation along the temporal direction, evidenced by row (c) in Fig. 17. This temporal broadening is a clear consequence of the nonfactorability of the twin-beam correlation in space and time. In turn, this is a consequence of the nonfactorable character of phase matching: the phase-matching condition [see Eq. (18)]

$$
\Delta^{\mathrm{pdc}}(q, \Omega) \approx \Omega^{2} / \Omega_{D}^{2}-q^{2} / q_{D}^{2}=0 \leftrightarrow k_{1}^{\prime \prime} \Omega^{2}=q^{2} / k_{1}
$$

can indeed be read as a compensation of the temporal dispersion experienced by twin photons inside the PDC crystal due to diffraction. Only if the entrance face of the second crystal is placed exactly in the image plane of the first crystal does this compensation occur, and the biphoton correlation is a nearly transform-limited coherent sum of the phase-matched spectral modes.

On the contrary, if the second crystal is misplaced with respect to the image plane, free propagation deteriorates this exact compensation of dispersion and diffraction. By making the simple assumption that only phase-matched modes contribute to the coherent SFG component, (43b), we can 

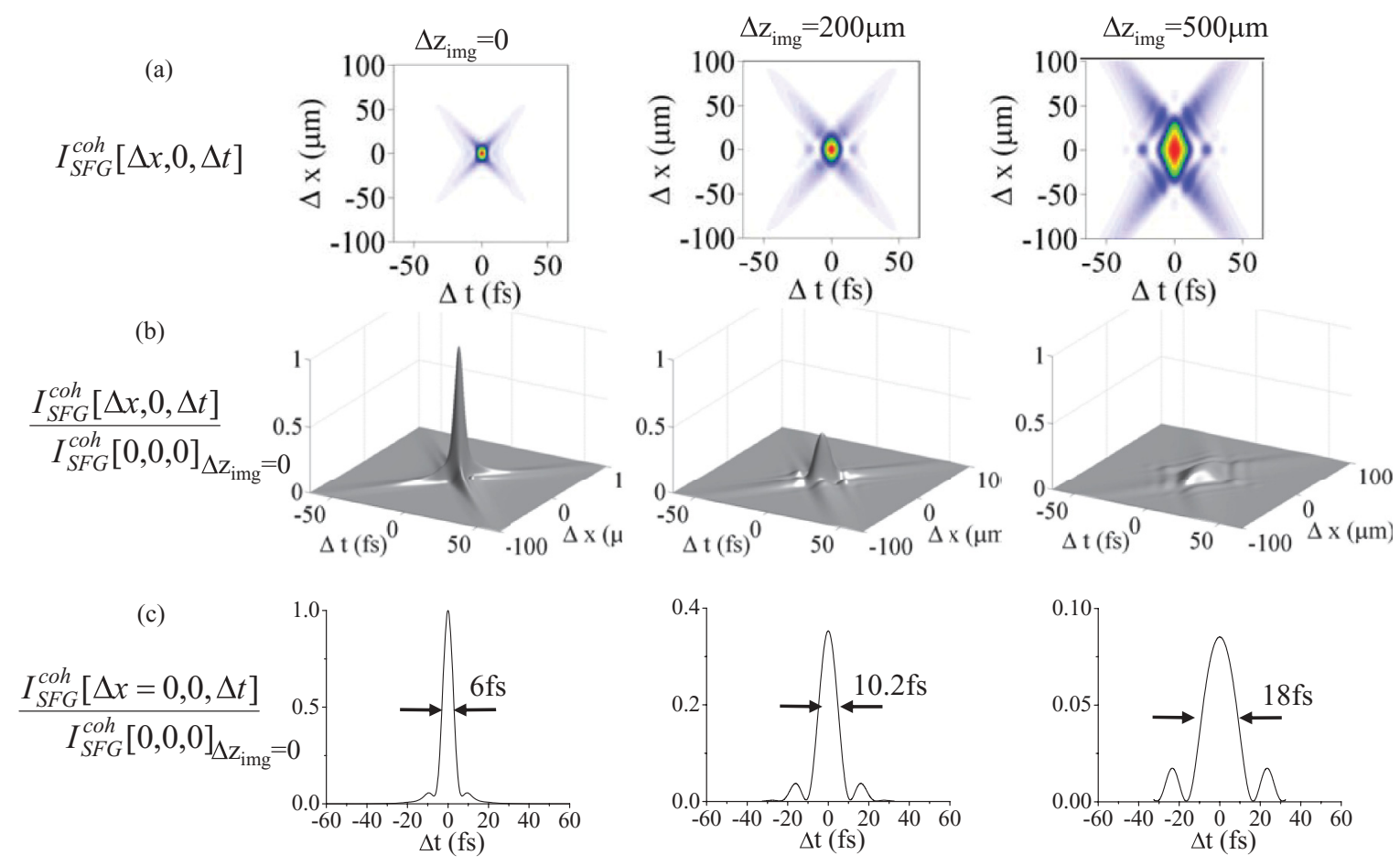

FIG. 17. (Color online) (a) Behavior of the reconstructed correlation $\left|\psi_{\text {meas }}(\Delta x, 0, \Delta t)\right|^{2}$, normalized to its peak value, for increasing values of $\Delta z_{\text {img }}$, showing the rapid deterioration of the X shape of the correlation. (b) Decrease in the peak height (normalized to the $\Delta z_{\text {img }}=0$ value). (c) Cross section of the central peak along the temporal delay $\Delta t$, evidencing a broadening of the temporal correlation for increasing errors in the imaging plane.

make the substitution $q^{2} \rightarrow \Omega^{2} k_{1} k_{1}^{\prime \prime}$ in the propagation term on the right-hand side of (57). It then transforms into the $\Omega$-dependent phase factor $e^{-i \frac{n_{1} k_{1}^{\prime \prime} \Omega^{2}}{1-\Omega^{2} / \omega_{1}^{2}} \Delta z_{\text {img }}}$, which describes a quadratic dispersion-like chirp of the twin beams.

\section{Effect of misalignments of the two crystals}

The coherent component of the SFG light is also strongly sensitive to misalignments of the SFG crystal with respect to the PDC crystal orientation. We investigated numerically the effect of a small tilt $\delta \theta_{0}^{\mathrm{SFG}} \neq 0$ of the SFG crystal with respect to the PDC crystal orientation, the latter satisfying perfect phase matching at degeneracy, i.e., $\Delta_{0}^{\mathrm{PDC}}=0$. Figure 18 shows how the peak of the coherent contribution, i.e., [see Eqs. (45) and (46)],

$$
I_{\mathrm{SFG}}^{\mathrm{coh}}[0,0,0]=\left|\int \frac{d \vec{w}}{(2 \pi)^{3}} F_{\mathrm{PDC}}(\vec{w}) F_{\mathrm{SFG}}^{(\mathrm{coh})}(\vec{w})\right|^{2},
$$

rapidly goes to zero as the tilt angle exceeds a few tenths of degrees. As expected, $I_{\mathrm{SFG}}^{(\mathrm{coh})}$ takes its maximum value when the two crystals are perfectly aligned, as $F_{\mathrm{PDC}}$ and $F_{\mathrm{SFG}}^{(\mathrm{coh})}$, and are peaked around the same phase-matching curves, and their overlap integral is maximized.

We can give a quantitative estimate of the angular tolerance in the alignment of the two crystals by considering explicitly the case when the second crystal is slightly tilted with respect to the first crystal, the latter being tuned for collinear phase matching. Denoting by $\theta^{\mathrm{PDC}}\left(\theta^{\mathrm{SFG}}\right)$ the orientation angle of the PDC (SFG) crystals with the pump axis (i.e., the $z$ axis), and assuming that the tilt angle between the two crystals $\delta \theta^{\mathrm{SFG}}=\theta^{\mathrm{SFG}}-\theta^{\mathrm{PDC}}$ is small, the following approximate relation between the pump-mode wave numbers in the two crystals holds:

$$
k_{0}^{\mathrm{SFG}} \approx k_{0}-k_{0} \rho_{0} \delta \theta^{\mathrm{SFG}},
$$

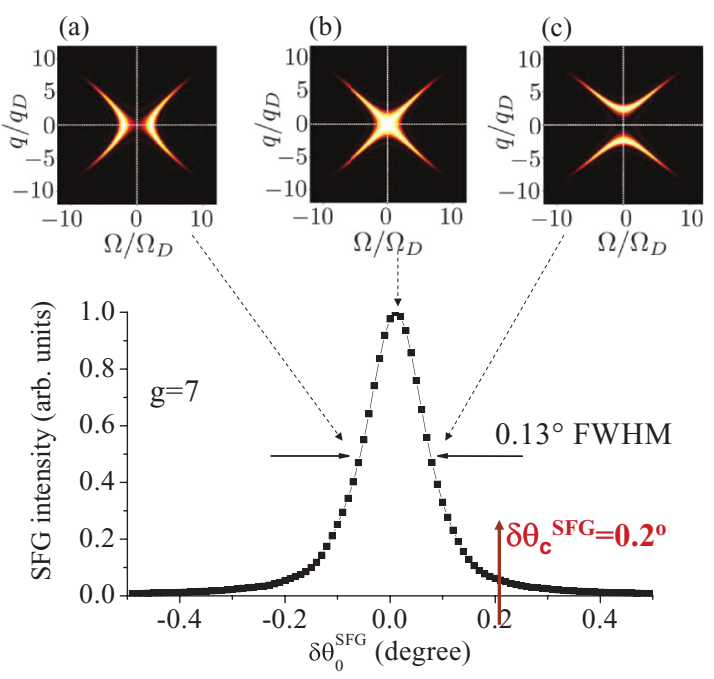

FIG. 18. (Color online) Bottom: Peak of the coherent SFG intensity $I_{\mathrm{SFG}}^{(\mathrm{coh})}[0,0,0]$ as a function of the angular tilt $\Delta \theta_{0}^{\mathrm{SFG}}$ between the two crystals (normalized to the value it has for $\delta \theta_{0}^{\mathrm{SFG}}=0$ ). Top: Plot of $\left|F_{\mathrm{SFG}}^{\text {(coh) }}(\vec{q}, \Omega)\right|^{2}$ for three orientations of the SFG crystal: (a) $\delta \theta_{0}^{\mathrm{SFG}}=-0.06^{\circ}, \Delta_{0}^{\mathrm{SFG}}=-4.24$; (b) $\delta \theta_{0}^{\mathrm{SFG}}=0^{\circ}, \Delta_{0}^{\mathrm{SFG}} l_{c}^{\prime}=0$; and (c) $\delta \theta_{0}^{\mathrm{SFG}} l_{c}^{\prime}=0.07^{\circ}, \Delta_{0}^{\mathrm{SFG}} l_{c}^{\prime}=5.8$. 
where $\rho_{0}=-\partial k_{0} /\left.\partial_{q_{x}}\right|_{\omega=\omega_{0}, q=0}$ is the positive defined walk-off angle of the extraordinary wave at frequency $\omega_{0}$. Since, by assumption, $\Delta_{0}^{\mathrm{PDC}} \equiv 2 k_{1}-k_{0}=0$, according to Eq. (61) a negative tilt $\delta \theta^{\mathrm{SFG}}<0$ leads to a positive collinear phase mismatch $\Delta_{0}^{\mathrm{SFG}} \equiv 2 k_{1}-k_{0}^{\mathrm{SFG}}=-k_{0} \rho_{0} \delta \theta^{\mathrm{SFG}}>0$ in the second crystal. At degeneracy the phase-matched spatial modes in the second crystal therefore lie on a circumference of radius

$$
q_{R}=q_{D} \sqrt{\Delta_{0}^{\mathrm{SFG}} l_{c}^{\prime}} \approx q_{D} \sqrt{k_{0} \rho_{0} l_{c}^{\prime}\left|\delta \theta^{\mathrm{SFG}}\right|} .
$$

Close to degeneracy, the region of overlap of $F_{\mathrm{PDC}}$ and $F_{\mathrm{SFG}}^{(\mathrm{coh})}$ can be expected to decrease drastically when $q_{R}$ coincides with the first node of the PDC probability amplitude $F_{\mathrm{PDC}}(\vec{q}, \Omega=0)$ along the spatial frequency axis, which is found to be well approximated by $\bar{q}_{D}=\sqrt{2}\left(\pi^{2}+g^{2}\right)^{1 / 4} q_{D}$ [see Eq. (20)]. Following this criterion, the overlap integral, (60), will be reduced by a large amount when the tilt angle exceeds the critical value

$$
\delta \theta_{c}^{\mathrm{SFG}}=\frac{2 \sqrt{\pi^{2}+g^{2}}}{\rho_{0} k_{0} l_{c}^{\prime}} .
$$

Taking, e.g., $g=7$, it gives us a tolerance $\delta \theta_{c}^{\mathrm{SFG}}=0.2^{\circ}$ in the alignment of the second $\mathrm{BBO}$ crystal with respect to the first. This value is in agreement with the numerical evaluation of $I_{\mathrm{SFG}}^{(\mathrm{coh})}[0,0,0]$ as a function of $\delta \theta_{0}^{\mathrm{SFG}}$ shown in Fig. 18.

\section{CONCLUSIONS}

Our treatment shows that the SFG process represents a powerful tool for exploring the biphotonic correlation in the full spatiotemporal domain. Through a careful manipulation of both the spatial and the temporal degrees of freedom of the PDC field emitted by the first $\chi^{(2)}$ crystal, the proposed optical setup allows us to retrieve the strongly localized $\mathrm{X}$-shaped PDC correlation in the space-time domain, predicted in Refs. [1]- [3]. The analytical result obtained within the PWPA shows that the coherent component of the up-converted SFG field contains the desired information on the PDC correlation function. In particular, this coherent component can be expressed in the form of a convolution between the PDC biphotonic function in direct space, the quantity under investigation, and the corresponding probability amplitude describing the up-conversion process in the SFG crystal, the convolution being evaluated at the applied temporal delay and spatial shift between two conjugate PDC components. It is shown that the measured quantity retains the main features of the biphotonic correlation, namely, its nonfactorable X-shaped geometry and its strong localization in space and time. The latter, which can, in principle, decrease to a few pump optical cycles [1], is determined by the acceptance bandwidth of the up-conversion process. Finally, a fully $3 \mathrm{D}+1$ numerical modeling of the optical setup that takes into account the pump pulse finite size has been implemented in order to provide a more realistic simulation of the experiment being implemented in Como. This allowed us to show that, even in the high-gain regime of PDC, the visibility of the correlation measurement can be close to $100 \%$ when the up-converted light is collected in the far field of the SFG crystal and in evaluation of the tolerance of the phase-sensitive correlation measurement against imperfection of the imaging system.

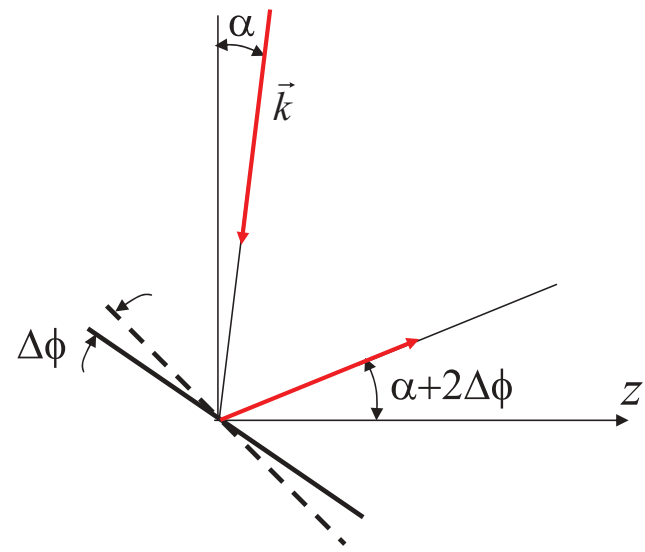

FIG. 19. (Color online) A rotation of $\Delta \phi$ of the mirror with respect to its original position (dashed line) generates a tilt by $2 \Delta \phi$ of the $k$ vector with respect to the $z$-axis direction after reflection.

\section{ACKNOWLEDGMENTS}

We are grateful to Paolo di Trapani for valuable suggestions and discussions. This work was realized in the framework of the Fet Open project of EC 221906 HIDEAS.

\section{APPENDIX: DERIVATION OF EQS. (26) AND (27)}

In this Appendix we describe the effect of a rotating mirror set in the $2 f$ plane of a $4 f$ telescopic system. Let us consider a rotation by $\Delta \phi$ of the mirror across the $y$ axis (orthogonal to the figure plane) with respect to its normal position set at $45^{\circ}$ with respect to the incident beam (dashed line in Fig. 19). Because of reflection, the $k$ vector of an incident mode $(\vec{q}, \Omega)$ with free-space wave number $k_{f}(\Omega)=\left(\omega_{1}+\Omega\right) / c$ and direction angle $\alpha=\arcsin \left(q_{x} / k_{f}\right)$ in the $(x, z)$ plane is tilted by an additional angle $2 \Delta \phi$ with respect to the $\Delta \phi=0$ direction. Accordingly, the $k$-vector transverse and longitudinal components in the plane orthogonal to the rotation axis, $q_{x}=k_{f} \sin \alpha$ and $k_{z}=k_{f} \cos \alpha$, transform according to the orthogonal transformations

$$
\begin{gathered}
q_{x}^{\prime}=q_{x} \cos 2 \Delta \phi+k_{f z} \sin 2 \Delta \phi, \\
k_{f z}^{\prime}=k_{f z} \cos 2 \Delta \phi-q_{x} \sin 2 \Delta \phi .
\end{gathered}
$$

For small angles, i.e., for $q_{x} / k_{f}=\sin \alpha \ll 1$ and $\Delta \phi \ll 1$, relations (A1) reduce to

$$
q_{x}^{\prime}=q_{x}+2 k_{f} \Delta \phi, \quad k_{f z}^{\prime}=k_{f z},
$$

where all powers of $q_{x} / k_{z}$ and $\Delta \phi$ have been neglected except the linear terms. Under this approximation the relation between the reflected and the incident field, $e_{1 r}$ and $e_{1 i}$, can be written as

$$
e_{1 r}\left(q_{x}, q_{y}, \Omega\right)=e_{1 i}\left(q_{x}-2 k_{f}(\Omega) \Delta \phi, q_{y}, \Omega\right) .
$$

The field Fourier transformation performed by the second lens of the $4 f$ system (see Fig. 3) can be written as

$$
\begin{aligned}
c_{1}(\vec{x}, \Omega) & =-\frac{i k_{f}(\Omega)}{2 \pi f} \int d \vec{x}^{\prime} e^{-i \frac{k_{f}(\Omega)}{f} \vec{x} \cdot \vec{x}^{\prime}} e_{1 r}(\vec{x}, \Omega) \\
& =-\frac{i k_{f}(\Omega)}{f} e_{1 r}\left(\vec{q}=\frac{k_{f}(\Omega)}{f} \vec{x}, \Omega\right)
\end{aligned}
$$


while that performed by the first lens can be written as

$$
e_{1 i}(\vec{q}, \Omega)=-\frac{i f}{k_{f}} b_{1}\left(\vec{x}=-\frac{f}{k_{f}} \vec{q}, \Omega\right) .
$$

Combining Eqs. (A3)-(A5), we readily obtain the following transformation between the input and the output planes of the telescopic system:

$$
c_{1}(\vec{x}, \Omega)=-b_{1}(-\vec{x}+\Delta \vec{x}, \Omega),
$$

where $\Delta \vec{x}=(2 f \Delta \phi, 0)$ denotes the transverse displacement of the field at the SFG crystal input plane produced by the mirror rotation. It is worth noting that, under this approximation, the transverse shift is the same at all temporal frequencies.

In the Fourier domain, Eq. (A6) reduces to the linear phaseshift transformation

$$
c_{1}(\vec{q}, \Omega)=-e^{i \vec{q} \cdot \Delta \vec{x}} b_{1}(-\vec{q}, \Omega)
$$

which coincides with input-output relation, (25), affecting the $q_{x}>0$ modes of beam + , except for the $\vec{q} \rightarrow-\vec{q}$ reflection and the -1 factor introduced by the two lenses, which has been omitted in the main treatment.
[1] A. Gatti, E. Brambilla, L. Caspani, O. Jedrkiewicz, and L. A. Lugiato, Phys. Rev. Lett. 102, 223601 (2009).

[2] L. Caspani, E. Brambilla, and A. Gatti, Phys. Rev. A 81, 033808 (2010).

[3] E. Brambilla, L. Caspani, L. A. Lugiato, and A. Gatti, Phys. Rev. A 82, 013835 (2010).

[4] B. E. A. Saleh, A. Joobeur, and M. C. Teich, Phys. Rev. A 57, 3991 (1998).

[5] C. Osorio, A. I. Valencia, and J. P. Torres, New J. Phys. 10, 113012 (2008).

[6] A. Gatti, R. Zambrini, M. San Miguel, and L. A. Lugiato, Phys. Rev. A 68, 053807 (2003).

[7] M. Atatüre, G. Di Giuseppe, M. D. Shaw, A. V. Sergienko, B. E. A. Saleh, and M. C. Teich, Phys. Rev. A 65, 023808 (2002); 66, 023822 (2002).

[8] M. Karpinski, C. Radzewicz, and K. Banaszek, Opt. Lett. 37, 878 (2012).

[9] P. J. Mosley, A. Christ, A. Eckstein, and C. Silberhorn, Phys. Rev. Lett. 103, 233901 (2009).

[10] O. Jedrkiewicz, J.-L. Blanchet, A. Gatti, E. Brambilla and P. Di Trapani, Opt. Express 19, 12903 (2011).

[11] O. Jedrkiewicz, J.-L. Blanchet, E. Brambilla, P. Di Trapani, and A. Gatti, arXiv:1203.3661v1 [Phys. Rev. Lett., in press (2012)].
[12] O. Jedrkiewicz, J.-L. Blanchet, E. Brambilla, O. Jedrkiewicz, L. A. Lugiato, and A. Gatti (in preparation).

[13] C. K. Hong, Z. Y. Ou, and L. Mandel, Phys. Rev. Lett. 59, 2044 (1987).

[14] B. Dayan, A. Peer, A. A. Friesem, and Y. Silberberg, Phys. Rev. Lett. 94, 043602 (2005).

[15] A. Peer, B. Dayan, A. A. Friesem, and Y. Silberberg, Phys. Rev. Lett. 94, 073601 (2005).

[16] S. E. Harris, Phys. Rev. Lett. 98, 063602 (2007).

[17] K. A. O'Donnell and A. B. U'Ren, Phys. Rev. Lett. 103, 123602 (2009).

[18] B. Dayan, Phys. Rev. A 76, 043813 (2007).

[19] B. Dayan, A. Peer, A. A. Friesem, and Y. Silberberg, Phys. Rev. Lett. 93, 023005 (2004).

[20] E. Brambilla, O. Jedrkiewicz, and A. Gatti (in preparation, 2012).

[21] E. Brambilla, A. Gatti, M. Bache, and L. A. Lugiato, Phys. Rev. A 69, 023802 (2004).

[22] V. G. Dmitriev, G., G. Gurzadyan, D. N. Nikogosyan, Handbook of Nonlinear Optical Crystals, Springer Series in Optical Sciences (Springer-Verlag, Berlin, 1991); N. Boeuf et al., Opt. Eng. 39, 1016 (2000). 